\title{
Concentration fluctuations in non-isothermal reaction-diffusion systems. II. The nonlinear case
}

\author{
D. Bedeaux, ${ }^{1, a)}$ J. M. Ortiz de Zárate, ${ }^{2, b)}$ I. Pagonabarraga, ${ }^{3}$ J. V. Sengers, ${ }^{4}$ \\ and S. Kjelstrup ${ }^{1,5}$ \\ ${ }^{1}$ Department of Chemistry, Norwegian University of Science and Technology, Trondheim 7491, Norway \\ ${ }^{2}$ Departamento de Física Aplicada I, Universidad Complutense, 28040 Madrid, Spain \\ ${ }^{3}$ Departamento de Fisica Fonamental, Universitat de Barcelona, 08028 Barcelona, Spain \\ ${ }^{4}$ Institute for Physical Science and Technology, University of Maryland, College Park, Maryland 20742, USA \\ ${ }^{5}$ Department of Process and Energy, Technical University Delft, 2628 CA Delft, The Netherlands
}

(Received 13 June 2011; accepted 29 August 2011; published online 30 September 2011)

\begin{abstract}
In this paper, we consider a simple reaction-diffusion system, namely, a binary fluid mixture with an association-dissociation reaction between two species. We study fluctuations at hydrodynamic spatiotemporal scales when this mixture is driven out of equilibrium by the presence of a temperature gradient, while still being far away from any chemical instability. This study extends the analysis in our first paper on the subject [J. M. Ortiz de Zárate, J. V. Sengers, D. Bedeaux, and S. Kjelstrup, J. Chem. Phys. 127, 034501 (2007)], where we considered fluctuations in a non-isothermal reactiondiffusion system but still close to equilibrium. The present extension is based on mesoscopic nonequilibrium thermodynamics that we recently developed [D. Bedeaux, I. Pagonabarraga, J. M. Ortiz de Zárate, J. V. Sengers, and S. Kjelstrup, Phys. Chem. Chem. Phys. 12, 12780 (2010)] to derive the law of mass action and fluctuation-dissipation theorems for the random contributions to the dissipative fluxes in the nonlinear macroscopic description. Just as for non-equilibrium fluctuations close to equilibrium, we again find an enhancement of the intensity of the concentration fluctuations in the presence of a temperature gradient. The non-equilibrium concentration fluctuations are in both cases spatially long ranged, with an intensity depending on the wave number $q$. The intensity exhibits a crossover from a $\propto q^{-4}$ to a $\propto q^{-2}$ behavior depending on whether the corresponding wavelength is smaller or larger than the penetration depth of the reacting mixture. This opens a possibility to distinguish between diffusion- or activation-controlled regimes of the reaction experimentally. The important conclusion overall is that non-equilibrium fluctuations in non-isothermal reaction-diffusion systems are always long ranged. (C) 2011 American Institute of Physics. [doi:10.1063/1.3640010]
\end{abstract}

\section{INTRODUCTION}

Although isothermal reaction-diffusion problems have been thoroughly studied in the scientific literature, reactiondiffusion in the presence of temperature gradients has received, comparatively, much less attention. This situation is a bit awkward because a chemical reaction very seldom takes place in a truly isothermal environment. Furthermore, it is well known that temperature gradients greatly affect transport processes in mixtures, and the importance of thermal diffusion (Soret effect) has been increasingly acknowledged during the last decades. ${ }^{1}$ Part of the problem is the different levels of description usually adopted for thermal diffusion and for chemical reactions. Specifically, the Soret effect is usually described in the phenomenological context of non-equilibrium thermodynamics, and good microscopic theories are lacking (except, may be, for the case of dilute gases). Chemical reactions, in contrast, are usually described by kinetic models, the current consensus being that thermodynamic models are only valid for chemical reactions at, or extremely close to, equilibrium. It is our opinion that the paucity of studies on nonisothermal reaction-diffusion can be overcome only when a

\footnotetext{
a)Electronic mail: dick.bedeaux@chem.ntnu.no.

b) Electronic mail: jmortizz@ fis.ucm.es.
}

common theoretical ground is established for both thermal diffusion and chemical reactions.

Non-isothermal reaction-diffusion systems are important in industry, as most chemical process plants contain units where such processes take place., ${ }^{2,3}$ These units, being out of global equilibrium, will experience hydrodynamic fluctuations. Such fluctuations arise from a coupling between velocity fluctuations and temperature and/or concentration fluctuations. As we shall demonstrate, these non-equilibrium fluctuations differ from fluctuations in equilibrium states by being spatially long ranged.

The traditional approaches for dealing with fluctuations in chemically reacting systems have been reviewed by Keizer. ${ }^{4}$ Most early attempts of extending these theories to non-equilibrium non-homogeneous states have implicitly assumed local equilibrium for the thermal thermodynamic fluctuations. ${ }^{5,6}$ However, in the absence of chemical reactions, experiments in fluids and fluid mixtures subjected to temperature and/or concentration gradient(s) have clearly demonstrated that the assumption of local equilibrium for the thermal fluctuations is not valid. ${ }^{7}$ Another recent and popular method to numerically study fluctuations in chemically reacting mixtures is the Gillespie algorithm, ${ }^{8}$ which is based on a master equation. This procedure accounts 
for fluctuations beyond the Gaussian approximation due to the finite number of reacting molecules and has been generalized to analyze a large variety of chemical reactions. ${ }^{9}$ This approach has also been extended to analyze inhomogeneous (but still isothermal) systems, where chemical kinetics competes with diffusion ${ }^{10}$ and to study fluctuations in spatially homogeneous systems near chemical instabilities. ${ }^{11,12}$ However, to our knowledge, a study based on the Gillespie algorithm ${ }^{8}$ of the spatial long-ranged nature of fluctuations in isothermal (but non-equilibrium) reaction-diffusion is not yet available; nor an extension of the algorithm itself to non-isothermal problems.

In the first paper, ${ }^{13}$ we derived expressions for the longrange hydrodynamic fluctuations of the concentration in a chemical reaction in a temperature gradient. The analysis was restricted to systems close to chemical equilibrium for which it was sufficient to use so-called linear kinetics for the chemical reactions, namely,

$$
r=-L_{r} \frac{\Delta g}{T},
$$

where $r$ is the chemical reaction rate, $L_{r}$ is a proportionality constant, and $T$ and $\Delta g$ denote the local values of, respectively, temperature and the chemical potential (or specific Gibbs energy) difference between products and reactants of the reaction, a quantity sometimes referred to as affinity. It is important to extend this analysis to realistic systems in which the chemical reaction is not close to equilibrium. For this purpose, we recently showed ${ }^{14}$ how the concept of mesoscopic non-equilibrium thermodynamics can be used to derive the law of mass action as well as to give fluctuationdissipation theorems for the random contributions to the dissipative fluxes on this level. In this paper, we will use these results to generalize the results found in the first paper ${ }^{13}$ to more realistic non-isothermal reaction-diffusion systems for which the simple linear approximation (1) is no longer valid.

That is, Eq. (1) cannot represent a kinetic law valid in general. In particular, it is not compatible with the wellaccepted kinetic law of mass action, the building block of chemical kinetics. This is a very well-known flaw of classical non-equilibrium thermodynamics. Inspired by Kramers' 15 study of a Brownian particle diffusing over a potential-energy barrier, a similar approach was suggested to build a nonequilibrium thermodynamics of chemical reactions. ${ }^{16,17}$ The basic idea is that one can imagine a chemical reaction as diffusion along a mesoscopic "internal" coordinate $\gamma$, from a reactant state to a product state. Such an internal diffusion process has to proceed over some barrier (in this case an enthalpy barrier). This analysis leads to a nonlinear relation between the local values of the chemical reaction rate $r$ and the affinity $\Delta g$, namely,

$$
r=-\frac{L_{r} R}{M}\left[1-\exp \left(-\frac{M \Delta g}{R T}\right)\right],
$$

where $M$ is the molar mass of the "reaction complex" (total molar mass of the reactants, that has to be equal to the total molar mass of the products) and $R$ is the ideal-gas constant. Equation (2) has enormous advantages over Eq. (1), the most important being its compatibility with the kinetic law of mass action. ${ }^{18}$ Notice that, when close to equilibrium, $M \Delta g \ll R T$ so that Eq. (2) reduces to Eq. (1).

Pagonabarraga et al. ${ }^{19}$ have shown how, using mesoscopic non-equilibrium thermodynamics in isothermal conditions, one obtains a chemical Langevin equation, where the statistical properties of the chemical noise are exactly the same as in the traditional, and more involved approach, that includes a chemical master equation, a Kramers-Moyal approximation and a van Kampen system-size expansion. ${ }^{20,21}$ Keizer $^{4}$ discussed the macroscopic equivalence of the chemical master equation with other approaches for the description of fluctuations in chemical reactions.

We recently ${ }^{14}$ started a program to develop mesoscopic non-equilibrium thermodynamics in the presence of a temperature gradient, so that the interplay between the chemical reaction, diffusion, and thermal diffusion can be described within the same theoretical framework. We considered fluctuations, and discussed how to deduce, on the basis of thermodynamics, the Langevin equation for the chemical reaction in the presence of a temperature gradient. However, we did not yet discuss the consequences of mesoscopic non-equilibrium thermodynamics for the fluctuations. That topic is addressed in the present paper in which we continue our previous study ${ }^{13}$ of temperature and concentration fluctuations in a reacting fluid mixture bounded between two plane parallel plates maintained at different temperatures. In our first paper, ${ }^{13}$ we also discussed the use of non-equilibrium molecular dynamics simulations for studies of transport phenomena. ${ }^{22,23}$

We present the balance equations and the transport equations for a simple reacting mixture in a temperature gradient in Sec. II. The description of fluctuations in the mixture and the relevant fluctuation-dissipation theorems are described in Sec. III. In the same section, it is explained why the fluctuations in equilibrium are the same as in the linear case. The application of the theory of fluctuations to a non-equilibrium system in a temperature gradient is presented in Sec. IV. The non-equilibrium fluctuations differ substantially from those at equilibrium. In Sec. V we discuss implications of our results for structure factors as measured in experiments. We summarize our main results and end with some concluding remarks in Sec. VI.

\section{NON-EQUILIBRIUM THERMODYNAMICS OF A CHEMICALLY REACTING FLUID MIXTURE}

A detailed treatment of chemically reacting fluid mixtures using mesoscopic non-equilibrium thermodynamics was presented by us in a recent publication. ${ }^{14}$ Using this mesoscopic description, we were able to derive the relevant nonlinear balance equations for the description on the macroscopic level. This macroscopic description contains random contributions to the dissipative fluxes. Fluctuation-dissipation theorems for these random contributions were given. In this section, we shortly review these equations.

As a representative example, we consider a reversible association-dissociation reaction, such as in a mixture of 
atoms and molecules,

$$
2 \mathrm{~A} \rightleftharpoons \mathrm{A}_{2} .
$$

For the particular case of fluorine atoms and molecules, the relevant transport properties are known from non-equilibrium molecular dynamics simulations. ${ }^{22,23}$ However, for our purpose, the detailed intermolecular interactions are not relevant and we treat Eq. (3) as a model chemical reaction to develop a theory of non-equilibrium concentration fluctuations that can be readily applied to any binary reaction-diffusion system. We shall assume that the kinetics of the chemical reaction (3) will be of the Kramers' type, Eq. (2).

The balance laws for the mass, momentum, and the internal-energy densities result in the following hydrodynamic equations:

$$
\begin{gathered}
\frac{D \rho}{D t}+\rho \nabla \cdot \mathbf{v}=0 \\
\rho \frac{D \mathbf{v}}{D t}=-\nabla p+\eta \nabla^{2} \mathbf{v}+\left(\frac{1}{3} \eta+\eta_{V}\right) \nabla \nabla \cdot \mathbf{v}, \\
\rho \frac{D c}{D t}=-L_{q J} \nabla^{2}\left(\frac{1}{T}\right)+L_{J J} \nabla^{2}\left(\frac{\Delta g}{T}\right) \\
-\frac{L_{r} R}{M}\left[1-\exp \left(-\frac{M \Delta g}{R T}\right)\right], \\
\rho c_{p} \frac{D T}{D t}-\alpha T \frac{D p}{D t}+\rho \Delta h \frac{D c}{D t} \\
=-L_{q q} \nabla^{2}\left(\frac{1}{T}\right)+L_{J q} \nabla^{2}\left(\frac{\Delta g}{T}\right),
\end{gathered}
$$

for the total mass density $\rho$, the barycentric velocity $\mathbf{v}$, the mass fraction $c$ of component $\mathrm{A}_{2}$, and the temperature $T$. In Eqs. (4), $c_{p}$ represents the isobaric specific heat capacity, $\eta$ is the shear viscosity, $\eta_{V}$ is the volume or bulk viscosity, $\alpha$ is the thermal-expansion coefficient, and $D / D t=\partial / \partial t+\mathbf{v} \cdot \nabla$ is the comoving time derivative. We suppress the dependence of the Onsager coefficients $L_{i j}$ on pressure, concentration, or temperature. Combined with the equations of state for the pressure $p=p(\rho, T, c)$ and for the Gibbs energy of the reaction $\Delta g=\Delta g(p, T, c)$, the above equations constitute the hydrodynamic equations of the chemically reacting mixture under consideration. The enthalpy of the chemical reaction is the difference $\Delta h=h_{\mathrm{A}_{2}}-h_{\mathrm{A}}$ between the enthalpies per unit mass of products and reactants. When $M \Delta g \ll R T$ the exponential function in Eq. (4c) may be linearized and the hydrodynamic equations then reduce to the ones given and used in our first paper. ${ }^{13}$

The deterministic steady-state solution is found by solving Eqs. (4) with the appropriate boundary conditions. This should be done before one calculates the fluctuations around such a solution. In a recent paper, ${ }^{24}$ we have discussed steadystate solutions in detail. For completeness, in Sec. IV A we give a short discussion of the procedure, with further details needed for the present paper summarized in the Appendix. The value of the various quantities in the deterministic solution are indicated by a subscript 0 .
Another ingredient we shall need here are the relations between the Onsager coefficients and the usual transport coefficients, that was clarified in our first paper, ${ }^{13}$

$$
\begin{aligned}
D & =\frac{L_{J J}}{\rho T}\left(\frac{\partial \Delta g}{\partial c}\right)_{p, T}, \quad \lambda=\frac{1}{T^{2}}\left[L_{q q}-\frac{L_{q J}^{2}}{L_{J J}}\right], \quad \text { (5) } \\
\rho D T k_{T} & =L_{q J}-L_{J J} \Delta h, \quad k_{p}=p\left(\frac{\partial \Delta g}{\partial p}\right)_{T, c}\left(\frac{\partial \Delta g}{\partial c}\right)_{p, T}^{-1},
\end{aligned}
$$

where $\lambda$ is the thermal conductivity, $D$ is the mutual diffusion coefficient, $k_{T}$ is a dimensionless thermal diffusion ratio, and $k_{p}$ is a dimensionless barodiffusion ratio. It is interesting to note that the barodiffusion ratio is independent of the Onsager coefficients; it is an equilibrium property and not related to a dissipative process. ${ }^{16,17}$ Barodiffusion seems to be important only in geological problems and is negligibly small for ordinary fluid mixtures. Hence, we neglect here barodiffusion, which means that we neglect the dependence of the specific Gibbs-energy difference on pressure, so that $\Delta g=\Delta g(T, c)$ only.

As the phenomenological coefficients for the energy and diffusion fluxes are related to the practical transport coefficients $D, \lambda$, and $k_{T}$, the coefficient $L_{r}$ associated with the chemical reaction can be related to the rate constants used in chemical kinetics. ${ }^{16,17,22,25}$ But this relationship is not as straightforward as for the other transport coefficients, since several complications in chemical kinetics ${ }^{26}$ need to be accounted for (concentrations units, distinction between reactions proceeding at constant volume or not, possibility of intermediate reaction steps, etc.). Hence, we prefer to use the Onsager coefficient $L_{r}$.

\section{FLUCTUATING HYDRODYNAMICS IN A CHEMICALLY REACTING MIXTURE}

Incorporation of the random contributions to the heat flux, $\delta \mathbf{J}_{q}(\mathbf{r}, t)$, diffusion flux, $\delta \mathbf{J}(\mathbf{r}, t)$, stress tensor, $\delta \Pi^{(\mathrm{st})}(\mathbf{r}, t)$, volume stress, $\delta \Pi(\mathbf{r}, t)$, and reaction rate, $\delta r(\mathbf{r}, t)$ in the hydrodynamic equations (4) yields ${ }^{14}$

$$
\begin{gathered}
\frac{D \rho}{D t}+\rho \nabla \cdot \mathbf{v}=0 \\
\rho \frac{D \mathbf{v}}{D t}=-\nabla p+\eta \nabla^{2} \mathbf{v}+\left(\frac{1}{3} \eta+\eta_{V}\right) \nabla \nabla \cdot \mathbf{v} \\
+\nabla \cdot \delta \Pi^{(\mathrm{st})}+\nabla \delta \Pi, \\
\rho \frac{D c}{D t}=-L_{q J} \nabla^{2}\left(\frac{1}{T}\right)+L_{J J} \nabla^{2}\left(\frac{\Delta g}{T}\right) \\
-\frac{L_{r} R}{M}\left[1-\exp \left(-\frac{M \Delta g}{R T}\right)\right]-\nabla \cdot \delta \mathbf{J}+\delta r \\
\rho c_{p} \frac{D T}{D t}-\alpha T \frac{D p}{D t}+\rho \Delta h \frac{D c}{D t} \\
=-L_{q q} \nabla^{2}\left(\frac{1}{T}\right)+L_{J q} \nabla^{2}\left(\frac{\Delta g}{T}\right)-\nabla \cdot \delta \mathbf{J}_{q} .
\end{gathered}
$$


The fluctuations around the deterministic solution are generally small. It is, therefore, appropriate to split the thermodynamic fields into deterministic parts $\left(p_{0}(\mathbf{r}, t), T_{0}(\mathbf{r}, t)\right.$ and $\left.c_{0}(\mathbf{r}, t)\right)$, and random fluctuating parts $\delta p(\mathbf{r}, t), \delta T(\mathbf{r}, t)$ and $\delta c(\mathbf{r}, t)$, which are driven by the random fluxes. We then obtain evolution equations for the thermodynamic fluctuations that include random forcing from the stochastic part of the fluxes. Since the fluctuations are small, the equations can be linearized in the fluctuating contributions. These random contributions to the macroscopic fluxes are all determined by Gaussian stochastic processes. It is, therefore, sufficient to give the first two moments of these contributions. The first moments are zero,

$$
\begin{aligned}
\left\langle\delta \mathbf{J}_{q}(\mathbf{r}, t)\right\rangle & =\langle\delta \mathbf{J}(\mathbf{r}, t)\rangle=\left\langle\delta \Pi^{(\mathrm{st})}(\mathbf{r}, t)\right\rangle \\
& =\langle\delta \Pi(\mathbf{r}, t)\rangle=\langle\delta r(\mathbf{r}, t)\rangle=0 .
\end{aligned}
$$

The non-zero fluctuation-dissipation theorems give the second moments, they are ${ }^{27}$

$$
\left\langle\delta J_{q, k}^{*}(\mathbf{r}, t) \delta J_{q, l}\left(\mathbf{r}^{\prime}, t^{\prime}\right)\right\rangle=2 k_{\mathrm{B}} L_{q q, 0}(\mathbf{r}, t) \delta_{k l} \delta\left(t-t^{\prime}\right) \delta\left(\mathbf{r}-\mathbf{r}^{\prime}\right),
$$

$$
\left\langle\delta J_{k}^{*}(\mathbf{r}, t) \delta J_{l}\left(\mathbf{r}^{\prime}, t^{\prime}\right)\right\rangle=2 k_{\mathrm{B}} L_{J J, 0}(\mathbf{r}, t) \delta_{k l} \delta\left(t-t^{\prime}\right) \delta\left(\mathbf{r}-\mathbf{r}^{\prime}\right),
$$

$$
\begin{aligned}
\left\langle\delta J_{k}^{*}(\mathbf{r}, t) \delta J_{q, l}\left(\mathbf{r}^{\prime}, t^{\prime}\right)\right\rangle & =\left\langle\delta J_{q, k}^{*}(\mathbf{r}, t) \delta J_{l}\left(\mathbf{r}^{\prime}, t^{\prime}\right)\right\rangle \\
& =2 k_{\mathrm{B}} L_{J q, 0}(\mathbf{r}, t) \delta_{k l} \delta\left(t-t^{\prime}\right) \delta\left(\mathbf{r}-\mathbf{r}^{\prime}\right),
\end{aligned}
$$

$$
\begin{aligned}
\left\langle\delta \Pi_{i j}^{(\mathrm{st}) *}(\mathbf{r}, t) \delta \Pi_{k l}^{(\mathrm{st})}\left(\mathbf{r}^{\prime}, t^{\prime}\right)\right\rangle= & 2 k_{\mathrm{B}}(T \eta)_{0}(\mathbf{r}, t)\left[\delta_{i k} \delta_{j l}+\delta_{i l} \delta_{j k}\right. \\
& \left.-\frac{2}{3} \delta_{i j} \delta_{k l}\right] \delta\left(t-t^{\prime}\right) \delta\left(\mathbf{r}-\mathbf{r}^{\prime}\right)
\end{aligned}
$$

$$
\left\langle\delta \Pi^{*}(\mathbf{r}, t) \delta \Pi\left(\mathbf{r}^{\prime}, t^{\prime}\right)\right\rangle=2 k_{\mathrm{B}}\left(T \eta_{V}\right)_{0}(\mathbf{r}, t) \delta\left(t-t^{\prime}\right) \delta\left(\mathbf{r}-\mathbf{r}^{\prime}\right),
$$

$$
\begin{aligned}
\left\langle\delta r^{*}(\mathbf{r}, t) \delta r\left(\mathbf{r}^{\prime}, t^{\prime}\right)\right\rangle= & k_{\mathrm{B}} L_{r, 0}(\mathbf{r}, t) \\
& \times\left[1+\exp \left(-\frac{M \Delta g_{0}(\mathbf{r}, t)}{R T_{0}(\mathbf{r}, t)}\right)\right] \\
& \times \delta\left(t-t^{\prime}\right) \delta\left(\mathbf{r}-\mathbf{r}^{\prime}\right) .
\end{aligned}
$$

We recall that the Onsager coefficients for the stress tensor are proportional to $T \eta$ and $T \eta_{V}$, and note that the fluctuationdissipation theorem for the random stress, written as in Eq. (8d), clearly shows the symmetry under permutation of indices: $i$ by $j$ or $k$ by $l$. Since the development in this paper refers to fluctuations around a deterministic solution of the mixture, all thermophysical properties in the fluctuationdissipation theorems are to be evaluated at the pressure $p_{0}(\mathbf{r})$, temperature $T_{0}(\mathbf{r})$ and concentration $c_{0}(\mathbf{r})$ of the deterministic steady-state solution. The values of the Onsager coefficients for the deterministic solution depend on the local values of $p_{0}, T_{0}, c_{0}$ and depend, therefore, on $\mathbf{r}$. For more details and a derivation from the mesoscopic level, we refer to our previous publication. $^{14}$

The heat and diffusion fluxes are both vectorial in nature and as a consequence the random contributions to these fluxes are correlated, see Eq. (8c). The viscous stress tensor is the only traceless symmetric tensor and is, therefore, not correlated with the other random flux contributions. The volume stress and the reaction rate are both scalar and the random contributions could, therefore, be correlated. This phenomenon is referred to as chemical viscosity. ${ }^{28}$ As this phenomenon is usually very small, ${ }^{28}$ we have neglected it.

In principle, the hydrodynamic fluctuations can be analyzed on the basis of the full set of hydrodynamic equations, as has been elucidated by Lekkerkerker and Laidlaw ${ }^{29}$ for fluctuations around an equilibrium state. However, for the sake of simplicity and to concentrate on the most salient physical features of our problem, we adopt the incompressibility approximation. This approximation is commonly made in dealing with fluctuations and with the onset of convection in liquid mixtures in the absence of a chemical reaction. ${ }^{7}$ In our particular case, ${ }^{30}$ the incompressibility approximation implies that we can neglect $\partial \rho / \partial t$ and $\nabla \rho$ in the first hydrodynamic equation, Eq. (4a) or Eq. (6b), as well as the term containing the thermal expansion $\alpha$ in the fourth, Eq. (4d) or Eq. (6d), while the mass density everywhere in the equations can be identified with an average uniform value $\rho=\rho_{0}$. The incompressibility assumption is equivalent to the assumption, usually adopted in chemical kinetics, ${ }^{26}$ that chemical reactions proceed at constant volume. Then, the hydrodynamic equations relevant to our current problem reduce to

$$
\begin{gathered}
\nabla \cdot \mathbf{v}=0 \\
\rho \frac{D \mathbf{v}}{D t}=-\nabla p+\eta \nabla^{2} \mathbf{v}+\nabla \cdot \delta \Pi^{(\mathrm{st})}, \\
\rho \frac{D c}{D t}=-L_{q J} \nabla^{2}\left(\frac{1}{T}\right)+L_{J J} \nabla^{2}\left(\frac{\Delta g}{T}\right) \\
-\frac{L_{r} R}{M}\left[1-\exp \left(-\frac{M \Delta g}{R T}\right)\right]-\nabla \cdot \delta \mathbf{J}+\delta r \\
\rho c_{p} \frac{D T}{D t}+\rho \Delta h \frac{D c}{D t} \\
=-L_{q q} \nabla^{2}\left(\frac{1}{T}\right)+L_{J q} \nabla^{2}\left(\frac{\Delta g}{T}\right)-\nabla \cdot \delta \mathbf{J}_{q} .
\end{gathered}
$$

An important simplification is that the velocity and pressure can be calculated from the first pair of equations without the need for using the other two. These solutions can then be substituted in the third and the fourth equations in order to calculate the mass fraction and the temperature. 


\section{A. Fluctuations around equilibrium}

When the fluid mixture is in thermal equilibrium in the absence of a temperature gradient, the solution of the hydrodynamic equations is obviously given by zero velocity $\mathbf{v}=0$, uniform pressure, uniform temperature $T=T_{\mathrm{e}}$ (equal to the temperature of the walls), and uniform concentration $c_{\mathrm{e}}$. This concentration $c_{\mathrm{e}}$ is the solution of the chemical equilibrium equation $\Delta g\left(c_{\mathrm{e}}, T_{\mathrm{e}}\right)=0$. The fluctuations around this equilibrium state are small and one may, therefore, use a completely linearized description of these fluctuations to calculate them. This we have in fact already done in our first paper ${ }^{13}$ to which we refer for a further discussion.

\section{FLUCTUATIONS IN A NON-ISOTHERMAL CHEMICALLY REACTING MIXTURE}

In the presence of a temperature difference, that is assumed to be stationary, the chemically reacting mixture will evolve to a non-equilibrium steady state. Our next goal is to evaluate the intensity of the fluctuations around such a nonequilibrium state. We start by discussing the stationary (deterministic) solution, about which many details are given in the Appendix. We follow here de Groot and Mazur ${ }^{16,17}$ by considering the stationary state of a chemically reacting fluid mixture in a temperature gradient enclosed in a reservoir, for which we can neglect the center-of-gravity motion $\left(\mathbf{v}_{0}=0\right)$. Note that we are neglecting buoyancy, so that any thermal convection is absent.

\section{A. The deterministic stationary solution}

We assume the presence of a deterministic temperature profile $T_{0}(x)$ in the $x$-direction, such that at $x=0$ the temperature $T_{0}(0)=T_{1}$ and at $x=L$ the temperature $T_{0}(L)=T_{L}$. Just as de Groot and Mazur, ${ }^{16,17}$ we consider the stationary solution with $\mathbf{v}_{0}=0$ and uniform pressure and with $\mathbf{J}_{0}=0$ at the boundaries of the reservoir at $x=0$ and $x=L$ (impervious walls). The analytic steady-state solution to this problem for the case of linear kinetics, Eq. (1), was reviewed by de Groot and Mazur, ${ }^{16,17}$ and recently revisited by some of us. ${ }^{13}$ Our goal here is to study the case of nonlinear Kramers kinetics, Eq. (2). If we impose a stationary (time-independent) state in the deterministic Eqs. (4), we find that there exists a quiescent $\left(\mathbf{v}_{0}=0\right)$ solution that is translationally invariant in the $Y Z$-plane (i.e., that depends only on the $x$-coordinate, $T_{0}(x)$ and $\left.\Delta g_{0}(x)\right)$, such that

$$
\begin{gathered}
0=-L_{q J} \frac{d^{2}}{d x^{2}}\left(\frac{1}{T_{0}}\right)+L_{J J} \frac{d^{2}}{d x^{2}}\left(\frac{\Delta g_{0}}{T_{0}}\right) \\
-\frac{L_{r} R}{M}\left[1-\exp \left(-\frac{M \Delta g_{0}}{R T_{0}}\right)\right], \\
0=-L_{q q} \frac{d^{2}}{d x^{2}}\left(\frac{1}{T_{0}}\right)+L_{J q} \frac{d^{2}}{d x^{2}}\left(\frac{\Delta g_{0}}{T_{0}}\right),
\end{gathered}
$$

where $L_{J J}, L_{q q}, L_{J q}=L_{q J}$ and $L_{r}$ represent the different Onsager transport coefficients, that we shall assume to be constants. As mentioned earlier, full compatibility with the law

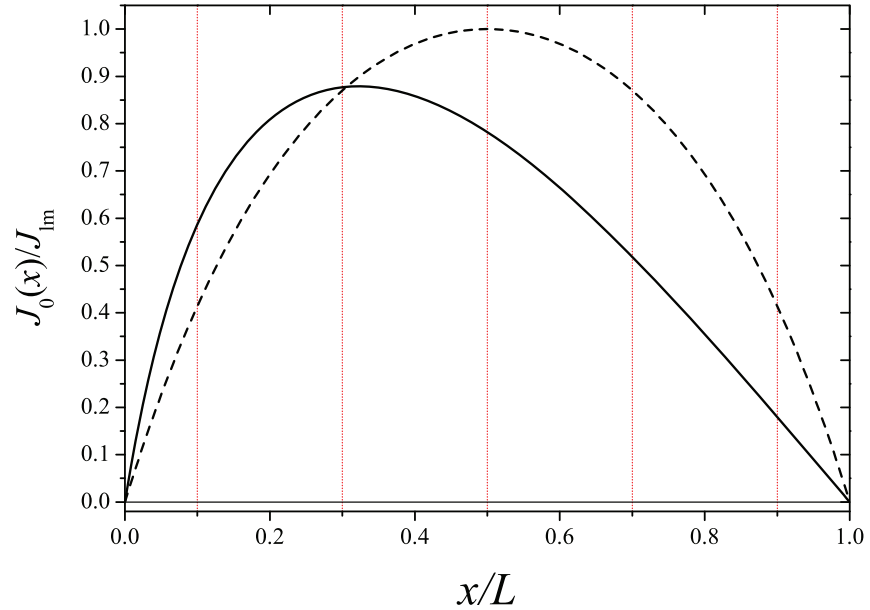

FIG. 1. Stationary flow profile $J_{0}(x)$, solution of Eqs. (10), as a function of $x$ (solid curve), compared with the profile corresponding to linear kinetics with the same values of the Onsager coefficients and $L_{r}$ (dashed curve). Data are normalized by the maximum flow in the linear case, $J_{\mathrm{lm}}$. Heating is from $x=0$ and $L_{J q}$ is positive. Data are for a fast chemical reaction and a large Lewis number (see main text).

of mass action requires $L_{r}$ in Eq. (2) to be a function of the concentration $c$. However, the effects of such extra nonlinearities are expected to be negligibly small. Our purpose here is to evaluate a minimal thermodynamic nonlinear model that is grosso modo compatible with the kinetic law of mass action. That is, we adopt Kramer's model, Eq. (2), with a constant coefficient $L_{r}$.

The solution $T_{0}(x)$ and $\Delta g_{0}(x)$ to Eqs. (10) satisfying the appropriate boundary conditions can be expressed in a semianalytical way, as discussed in another publication, ${ }^{24}$ where some of the most salient features of this nonlinear solution, in particular the influence of the Soret effect as compared with the classical linear solution reviewed by de Groot and Mazur, ${ }^{13,16,17}$ were discussed. As already mentioned, some details of such a nonlinear solution procedure ${ }^{24}$ are summarized in the Appendix.

For our purposes, here it is most important to discuss the deterministic stationary diffusion flow profile, $J_{0}(x)$, for which an analytic relationship exist with the ratio $\Delta g_{0}(x) / T_{0}(x)$, Eqs. (A6) or (A8) in the Appendix. A typical $J_{0}(x)$ is shown in Fig. 1, that corresponds to heating from $x=0$ and positive Soret effect. ${ }^{24}$ Parameter values in Fig. 1 are $\phi=L / d=7$, with $d$ the penetration depth of the chemical reaction as defined by Eq. (A3) in the Appendix; and $b / a \phi=15$. The former indicates a fast chemical reaction, while the latter indicates a large Lewis number, see Eqs. (A12) and (A13) and the subsequent discussion in the Appendix.

One observes in the figure that, as required by the boundary conditions of impervious walls, $J_{0}(x)$ goes to zero at both $x=0$ and $x=L$. In between, it presents a maximum at a position $x_{\mathrm{m}}$ that, in general, differs from $L / 2$. It is worth noticing that in the linear kinetics solution, reviewed by de Groot and Mazur, ${ }^{13,16,17}$ the maximum stationary diffusion flow is always located at $x=L / 2$, see dashed curve in Fig. 1 . This asymmetry in the flow profile is one of the most salient features of the stationary solution for nonlinear Kramers kinetics 


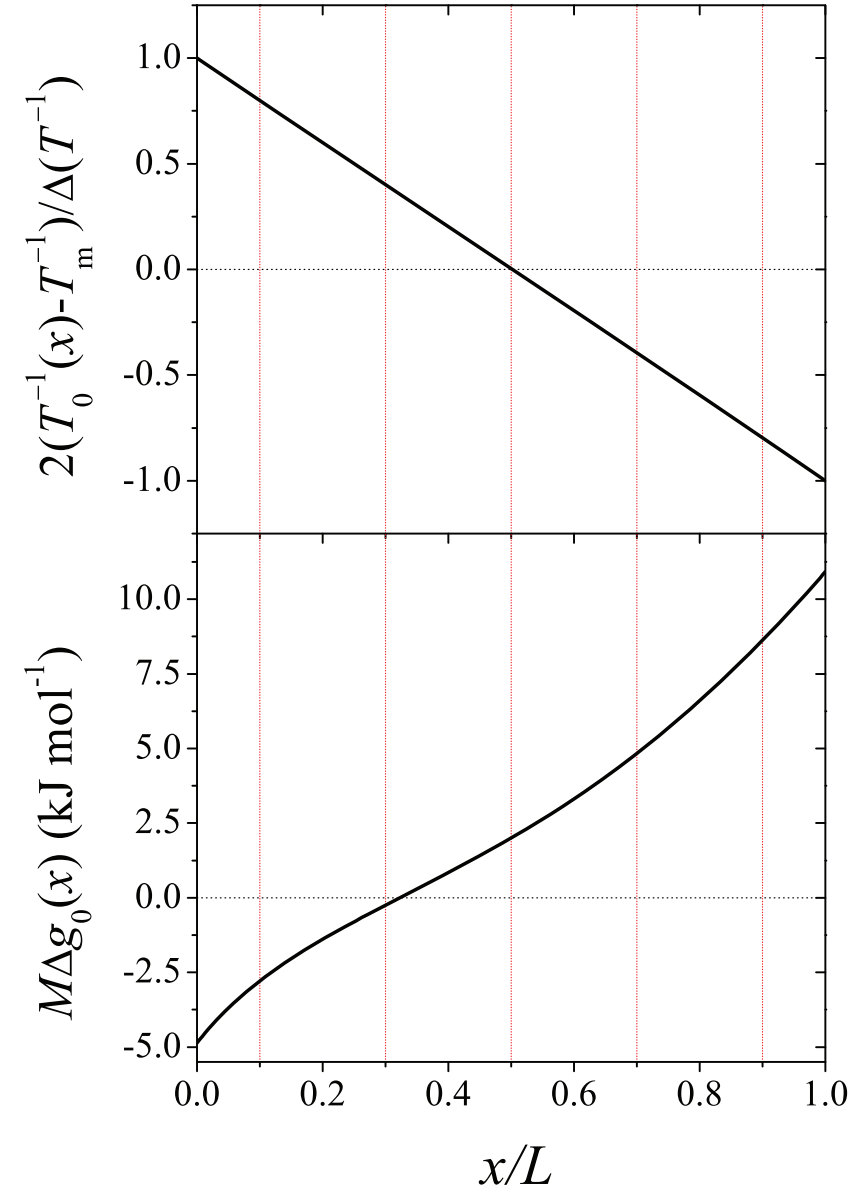

FIG. 2. Stationary profiles of inverse temperature (top panel) and $M \Delta g_{0}(x)$ (bottom panel), for the same set of parameters for which the diffusion profile was shown in Fig. 1 (as a solid curve). Top panel shows the difference between the local inverse temperature and the harmonic average of the boundary temperatures $T_{\mathrm{m}}^{-1}$, normalized by (half) the difference of inverse temperatures $\Delta\left(T^{-1}\right)=1 / T_{0}-1 / T_{L}$. For this example temperatures are $T_{0}$ $=320 \mathrm{~K}$ and $T_{L}=300 \mathrm{~K}$, and $x_{\mathrm{m}} / L \simeq 0.323$.

and depends on the sign of the Soret coefficient. ${ }^{24}$ For a positive Soret effect, $x_{\mathrm{m}}$ is closer to the hot plate, while for a negative Soret effect $x_{\mathrm{m}}$ is closer to the cold plate. See a more complete discussion in the Appendix.

From Eq. (A6) or Eq. (A8) in the Appendix, it follows that at the location $x_{\mathrm{m}}$ where the stationary diffusion flow is maximum,

$$
\exp \left(-\frac{M \Delta g_{\mathrm{m}}}{R T_{\mathrm{m}}}\right)=1
$$

or, alternatively, $\Delta g_{\mathrm{m}}=0$, as shown in the bottom panel of Fig. 2. From Eq. (2) it follows that, at the location of maximum stationary diffusion flow the chemical reaction rate is zero. We shall make use of these properties in the evaluation of the intensity of the concentration fluctuations. In what follows, the subscript $\mathrm{m}$ will indicate the value for the deterministic solution at position $x_{\mathrm{m}}$.

In general, the inverse temperature profiles also present asymmetry, and the midpoint of the layer is no longer at the harmonic average of the boundary temperatures, as occurs always for the case of linear kinetics. ${ }^{17}$ Furthermore, in general, the location of the temperature harmonic average does not coincide with the location of maximum diffusion flow. The difference between both locations can be related to the Lewis number. In the present paper, we only consider the stationary solution in the limit of very large Lewis number (see Sec. IV B). In this limit, one sees from Eq. (A12) in the Appendix that the parameter $a$ controlling the shape of the stationary temperature profile vanishes. As a consequence, in the large-Lewis-number limit the profile $1 / T_{0}(x)$ will simply proceed linearly from the inverse temperature at one of the boundaries $\left(1 / T_{0}\right)$ to the inverse temperature at the other boundary $\left(1 / T_{L}\right)$, as shown in the top panel of Fig. 2. In this particular case $(\mathrm{Le} \rightarrow \infty)$, the harmonic average of the boundary temperatures is located at the midpoint of the layer.

The concentration profiles $c(x)$ at large Lewis number can be obtained from the $M \Delta g_{0}(x)$ profiles, as the one shown in the bottom panel of Fig. 2. For this calculation knowledge of the equation of state $\Delta g(c, T)$ is required. If, for instance, one assumes a regular solution model for the equation of state, one obtains nonlinear asymmetric $c(x)$ profiles; with a general shape quite similar to the $M \Delta g_{0}(x)$ profile shown in the bottom panel of Fig. 2 but conveniently translated and rescaled.

In summary, systems with large Lewis number are characterized by noticeably nonlinear and asymmetric diffusion flow, $\Delta g_{0}(x)$ and concentration profiles, but an almost linear inverse-temperature profile. This difference between concentration and temperature profiles is expected because a large Lewis-number approximation implies the assumption that heat diffusion is infinitely faster than molecular diffusion. As further discussed in the Appendix, the inverse penetration depth $(\phi=0.143$ for the data in Figs. 1 and 2), see Eq. (A3), gives a rough idea of the region, close to the boundaries, where the gradient of $\Delta g_{0}$ is noticeably different from the gradient at the point of maximum flow.

\section{B. Equations for the fluctuations}

We start the calculation of the non-equilibrium structure factor of the fluid by setting up the evolution equations for the thermodynamic fluctuations around the stationary solution described in the previous subsection. We start from the balance laws in the form (9) and represent in the right-hand side (RHS) the thermodynamic fields by their stationary values plus some "fluctuation," for instance, $T(\mathbf{r}, t)$ $=T_{0}(x)+\delta T(\mathbf{r}, t), \mathbf{v}(\mathbf{r}, t)=\delta \mathbf{v}(\mathbf{r}, t)$, etc. This procedure yields fluctuating-hydrodynamics equations from which the spatiotemporal evolution of the fluctuating thermodynamic variables may be computed.

The generic procedure described above produces some complicated nonlinear equations and some approximations are of rigueur. First of all, some simplifications come from the observation that $T_{0}(x)$ and $\Delta g_{0}(x)$ are solutions of the stationary Eqs. (10). Next, the most important simplification is obtained by linearizing the resulting expressions in the fluctuating fields. This approximation is justified if the fluctuations are "small." Previous work on non-reacting mixtures has confirmed that this is the case when the stationary solution is stable, i.e., when the system is not close to a hydrodynamic instability such as the onset of convection. ${ }^{7}$ Here, we continue 
to proceed with the assumption that the stationary solution is stable, so that we can linearize our evolution equations for the non-equilibrium fluctuations. After having linearized the equations, a further simplification can be obtained by taking a double curl of the fluctuating Navier-Stokes equation and by using the divergence-free condition, $\nabla \cdot \delta \mathbf{v}=0$. This procedure decouples the equations for the fluctuations in the $y$ and $z$-components of the velocity from the temperature or concentration fluctuations. With these simplifications, the fluctuating hydrodynamic equations (expressed in terms of the ordinary transport coefficients related to Onsager's coefficients by Eqs. (5)) read as

$$
\begin{gathered}
\frac{\partial}{\partial t} \nabla^{2} \delta v_{x}=v \nabla^{2}\left(\nabla^{2} \delta v_{x}\right) \\
+\frac{1}{\rho}\left\{\nabla \times \nabla \times\left(\nabla \cdot \delta \Pi^{(\mathrm{st})}\right)\right\}_{x} \\
\frac{\partial}{\partial t} \delta c+\delta v_{x} \frac{d c_{0}}{d x}=D\left\{\nabla^{2} \delta c+\frac{k_{T}}{T_{0}} \nabla^{2} \delta T\right\}-\frac{L_{r}}{\rho} \\
\quad \times \exp \left(-\frac{M \Delta g_{0}}{R T_{0}}\right) \delta\left(\frac{\Delta g}{T}\right) \\
\frac{\partial}{\partial t} \delta T+\delta \mathbf{J}+\frac{1}{\rho} \delta r \\
=\left[a_{T}+D\left(\epsilon_{x} \frac{d T_{0}}{d x}+T_{0} \Delta \tilde{h}\left[\frac{\partial}{\partial t} \delta c+\delta v_{x} \frac{d c_{0}}{d x}\right]\right.\right. \\
\left.+\frac{D T_{0}}{k_{T}}\left(\epsilon_{\mathrm{D}}+k_{T} \Delta \tilde{h}\right)\right] \nabla^{2} \delta T
\end{gathered}
$$

where the subscript $x$ in Eq. (12a) indicates the $x$ component of the vector between curly brackets. In Eqs. (12), $a_{T}=\lambda / \rho c_{p}$ is the thermal diffusivity, $\epsilon_{\mathrm{D}}$ is the dimensionless Dufour effect ratio,

$$
\epsilon_{\mathrm{D}}=\frac{k_{T}^{2}}{c_{p} T}\left(\frac{\partial \Delta g}{\partial c}\right)_{p, T}
$$

and $\Delta \tilde{h}$ is the dimensionless specific enthalpy of reaction,

$$
\Delta \tilde{h}=\frac{\Delta h}{c_{p} T}=\frac{1}{c_{p} T}\left[\Delta g-T\left(\frac{\partial \Delta g}{\partial T}\right)_{p, c}\right] .
$$

We shall neglect the spatial dependence of these two last quantities; hence, in what follows we consider them as (uniform) average values through the fluid layer. We recall that due to the incompressibility assumption the density $\rho=\rho_{0}$ is uniform. Furthermore, we neglect throughout this paper, the position dependence of all thermophysical properties of the mixture, such as the Onsager coefficients and the transport coefficients. The influence of such nonlinearities in nonequilibrium fluctuations has been considered in Refs. 31 and 32 and is negligible when non-equilibrium coupling between the fluctuating fields exist, as is the case here. Furthermore, consistency with the steady-state solution described in Sec. IV A, also requires to consider here all transport coefficients (including reaction rate) as constants. In the linear case Eqs. (12) reduce to Eqs. (51) in our first paper. ${ }^{13}$
It is interesting to compare this equation with the one for the fluctuations in equilibrium, which are obtained from Eqs. (12) by setting $d c_{0} / d x=d T_{0} / d x=0$ and $\Delta g_{0}=0$. We notice that this eliminates the coupling between the velocity fluctuations parallel to the gradient and the temperature or concentration fluctuations. In non-equilibrium, velocity fluctuations in the direction of the stationary gradients probe regions with different temperatures and concentrations. As in the case of a non-isothermal non-reacting binary mixture, ${ }^{7}$ this coupling is precisely the origin of a large enhancement of the thermodynamic fluctuations when the system is in a non-equilibrium steady state. The nonlinear nature of the equation far from equilibrium leads to the additional factor $\exp \left(-M \Delta g_{0} / R T_{0}\right)$ in the term proportional to $L_{r, 0}$. This can lead to a substantial increase or decrease of this term compared to the linear case.

Although non-equilibrium fluctuations can be directly evaluated from Eqs. (12), again a further simplification can be achieved by exploiting the fact that for dense fluids (liquids) the Lewis number Le $=a_{T} / D$ is usually quite large. For non-equilibrium fluid mixtures, the large-Lewis-number approximation was first proposed by Velarde and Schechter ${ }^{33}$ so as to simplify the instability analysis when buoyancy effects are incorporated in the theory. The same approximation scheme has also been successfully used by some of us to study non-equilibrium concentration fluctuations induced by the Soret effect in a non-reacting binary mixture. ${ }^{34,35}$ The most important consequence of this large-Lewis-number approximation is that temperature fluctuations can be ignored, and that only the coupling between concentration and velocity fluctuations needs to be considered. Indeed, in ordinary liquid mixtures concentration fluctuations usually dominate, and temperature fluctuations are more difficult to observe by experimental techniques, such as light scattering. ${ }^{36-38}$ The same has been found from non-equilibrium molecular dynamics simulation. ${ }^{39}$ Further details concerning this approximation scheme can be found in the relevant literature. ${ }^{33,35}$ In the Le $\rightarrow \infty$ limit, the set of working Eqs. (12) reduces to

$$
0=v_{0} \nabla^{4} \delta v_{x}+\frac{1}{\rho_{0}}\left\{\nabla \times \nabla \times\left(\nabla \cdot \delta \Pi^{(\mathrm{st})}\right)\right\}_{x},
$$

$$
\begin{aligned}
\frac{\partial}{\partial t} \delta c & +\delta v_{x} \nabla c_{0} \\
= & D_{0} \nabla^{2} \delta c-\frac{L_{r, 0}}{\rho_{0} T_{0}} \exp \left(-\frac{M \Delta g_{0}}{R T_{0}}\right)\left(\frac{\partial \Delta g_{0}}{\partial c_{0}}\right)_{p, T} \delta c \\
& -\frac{1}{\rho_{0}} \nabla \cdot \delta \mathbf{J}+\frac{1}{\rho_{0}} \delta r .
\end{aligned}
$$

Note that far from equilibrium $\exp \left(-\left(M \Delta g_{0} / R T_{0}\right)\right)$ changes from a value much smaller than unity at one end of the box to a value much larger than unity at the other end of the box. It is this term that gives the major difference with the analysis of the fluctuations around a stationary solution that is everywhere close to chemical equilibrium. We expect that our present results may also be applicable to some kinds of open systems for which the stationary solution can still be represented by Eqs. (10) with $\mathbf{v}=0$ but with a non-vanishing diffusion flux $\mathbf{J}$ at the boundary. ${ }^{40}$ 
We shall not consider boundary conditions for the fluctuating fields in this paper. In general, fluctuations in fluids subjected to a temperature gradient at large wavelengths comparable to the size of the system are affected by the presence of boundaries. ${ }^{7}$ However, it has been experimentally shown that such boundary conditions are not needed to reproduce the proper asymptotic behavior of the non-equilibrium hydrodynamic fluctuations at small wavelengths ${ }^{41}$ (but still large enough to be in the hydrodynamic regime). Deviations from our solution are expected for larger wavelengths due to confinement effects, but they are not considered in the present paper.

\section{Procedure for solving fluctuating equations}

In matrix form, we may write Eqs. (15) as

$$
\begin{aligned}
& \left(\begin{array}{cc}
v_{0} \nabla^{4} & 0 \\
\nabla c_{0}(x) & \frac{\partial}{\partial t}+D_{0} \nabla^{2}-\frac{L_{r, 0} R}{\rho_{0} M}\left[\frac{\partial}{\partial c(x)} \exp \left(-\frac{M \Delta g(x)}{R T(x)}\right)\right]_{T, 0}
\end{array}\right) \cdot\left(\begin{array}{c}
\delta v_{x}(\mathbf{r}, t) \\
\delta c(\mathbf{r}, t)
\end{array}\right) \\
& =\frac{1}{\rho_{0}}\left(\begin{array}{c}
-\left\{\boldsymbol{\nabla} \times \nabla \times\left(\nabla \cdot \delta \Pi^{(\mathrm{st})}(\mathbf{r}, t)\right)\right\}_{x} \\
-\nabla \cdot \delta \mathbf{J}(\mathbf{r}, \mathbf{t})+\delta r(\mathbf{r}, t)
\end{array}\right) \equiv \mathbf{F}(t, \mathbf{r}) .
\end{aligned}
$$

Taking a full spatiotemporal Fourier transform of Eq. (16), we obtain

$$
\begin{aligned}
& \left(\frac{1}{2 \pi}\right)^{3} \int d \mathbf{q}^{\prime} \mathbf{G}^{-1}\left(\omega, \mathbf{q}, \mathbf{q}^{\prime}\right) \cdot\left(\begin{array}{c}
\delta v_{x}\left(\omega, \mathbf{q}^{\prime}\right) \\
\delta c\left(\omega, \mathbf{q}^{\prime}\right)
\end{array}\right) \\
& =\mathbf{F}(\omega, \mathbf{q})=\frac{1}{\rho_{0}}\left(\begin{array}{c}
i \epsilon_{x n i} q_{n} \epsilon_{i j k} q_{j}\left[q_{l} \delta \Pi_{l k}^{(\mathrm{st})}(\omega, \mathbf{q})\right] \\
-i q_{i} \delta J_{i}(\omega, \mathbf{q})+\delta r(\omega, \mathbf{q})
\end{array}\right),
\end{aligned}
$$

where $\omega$ and $\mathbf{q}$ are the frequency and the wave vector of the fluctuations, respectively. In the expression of the vector of random forces $\mathbf{F}(\omega, \mathbf{q})$, summation over repeated indices is understood, and in the first of the Levi-Civita tensors $\epsilon$, an index $x$ appears because the actual random force corresponds to the $x$-component of the vector between curly brackets in the RHS of Eq. (16). The explicit expression of the inverse linear response function for the non-equilibrium fluctuations on the LHS of Eq. (17) is

$$
\mathbf{G}^{-1}\left(\omega, \mathbf{q}, \mathbf{q}^{\prime}\right)=\left(\begin{array}{cc}
\nu_{0} q^{4}(2 \pi)^{3} \delta\left(\mathbf{q}-\mathbf{q}^{\prime}\right) & 0 \\
\left(\nabla c_{0}\right)\left(\mathbf{q}-\mathbf{q}^{\prime}\right) & -\frac{L_{r, 0} R}{\rho_{0} M} F\left[\frac{\partial \exp \left(-\frac{M \Delta g}{R T}\right)}{\partial c}\right]_{T, 0}\left(\mathbf{q}-\mathbf{q}^{\prime}\right)
\end{array}\right),
$$

where $F\left[\frac{\partial}{\partial c} \exp \left(-\frac{M \Delta g}{R T}\right)\right]_{T, 0}(q)$ is the Fourier transform of $\left[\frac{\partial}{\partial c(x)} \exp \left(-\frac{M \Delta g(x)}{R T(x)}\right)\right]_{T, 0}$. There are two terms in this expression for which we need a proper approximation. Let us first consider $\left[\frac{\partial}{\partial c(x)} \exp \left(-\frac{M \Delta g(x)}{R T(x)}\right)\right]_{T, 0}$. For an ideal system, the exponent is equal to $c_{0} /\left(1-c_{0}\right)^{2}$. This implies that the derivative with respect to $c_{0}$ is $\left(1+c_{0}\right) /\left(1-c_{0}\right)^{3}$. In the general case, there are activity corrections. Far from chemical equilibrium $M \Delta g / R T$ varies from a value much smaller than -1 to a value much larger than +1 from $x$
$=0$ to $x=L$. This implies that the concentration also varies substantially along the box. Close to equilibrium, it was sufficient to replace this term by its value midway between the two plates. ${ }^{13}$ Far from equilibrium, we need to take the variation from one end of the box to the other into account to see how the results of the linear approximation for the reaction rate are modified. As reference point, we take the location $x_{\mathrm{m}}$ at which the stationary diffusion flux was maximum, see Fig. 1. To lowest order in the variation we will, therefore, use 


$$
\begin{aligned}
F\left[\frac{\partial}{\partial c} \exp \left(-\frac{M \Delta g}{R T}\right)\right]_{T, 0}(\mathbf{q}) & =\int d \mathbf{r} \exp \left(i \mathbf{q} \cdot\left(x-x_{m}, y, z\right)\right)\left[\frac{\partial}{\partial c} \exp \left(-\frac{M \Delta g}{R T}\right)\right]_{T, 0}(\mathbf{r}) \\
& =\left[2 \pi F_{\mathrm{m}} \delta\left(q_{x}\right)-2 \pi i F_{1} \frac{\partial}{\partial q_{x}} \delta\left(q_{x}\right)\right](2 \pi)^{2} \delta\left(q_{y}\right) \delta\left(q_{z}\right),
\end{aligned}
$$

where

$$
F_{\mathrm{m}}=\left[\frac{\partial}{\partial c} \exp \left(-\frac{M \Delta g_{0}}{R T_{0}}\right)\right]_{\mathrm{m}}=-\frac{M}{R T_{\mathrm{m}}}\left(\frac{\partial \Delta g}{\partial c}\right)_{T, \mathrm{~m}}
$$

and

$$
\begin{aligned}
F_{1}= & \left\{\frac{\partial}{\partial x}\left[\frac{\partial}{\partial c} \exp \left(-\frac{M \Delta g}{R T}\right)\right]\right\}_{\mathrm{m}} \\
= & \frac{M}{R T_{\mathrm{m}}}\left\{\left[\frac{M}{R T}\left(\frac{\partial \Delta g}{\partial c}\right)^{2}-\frac{\partial^{2} \Delta g}{\partial c^{2}}\right]_{T} \nabla c\right. \\
& \left.-\frac{1}{T}\left[\frac{M \Delta h}{R T} \frac{\partial \Delta g}{\partial c}-\frac{\partial \Delta h}{\partial c}\right] \nabla T\right\}_{\mathrm{m}}
\end{aligned}
$$

As mentioned above, the subscript $m$ indicates the value for the deterministic solution at position $x_{\mathrm{m}}$. The knowledge of the equation of state, $\Delta g(c, T)$, is assumed and Eq. (11) has been employed to simplify the expressions. The inverse Fourier transform of Eq. (19) gives

$$
\left[\frac{\partial}{\partial c} \exp \left(-\frac{M \Delta g}{R T}\right)\right]_{T, 0}(\mathbf{r})=F_{\mathrm{m}}+F_{1}\left(x-x_{\mathrm{m}}\right) .
$$

We note that, in general, the point $x_{\mathrm{m}}$ differs only a little from the midpoint of the interval $L / 2$, see Fig. 1 for a typical example. As Eqs. (20a) and (20b) show, we simplify the description by taking the value of the concentration and its first derivative at the point $x_{\mathrm{m}}$ where the stationary diffusion flux $J_{0}(x)$ is maximum. The operator $\mathrm{G}^{-1}$ furthermore contains a term $\left(\nabla c_{0}\right)\left(\mathbf{q}-\mathbf{q}^{\prime}\right)$. This is already a first-order derivative of the density and we may, therefore, approximate this term by

$$
\left(\nabla c_{0}\right)\left(\mathbf{q}-\mathbf{q}^{\prime}\right)=(\nabla c)_{\mathrm{m}}(2 \pi)^{3} \delta\left(\mathbf{q}-\mathbf{q}^{\prime}\right) .
$$

Substituting Eqs. (19) and (22) into Eq. (18) gives

$$
\begin{aligned}
\mathrm{G}^{-1}\left(\omega, \mathbf{q}, \mathbf{q}^{\prime}\right) & \\
= & \left(\begin{array}{cc}
v_{0} q^{4} & 0 \\
(\nabla c)_{\mathrm{m}} & \mathrm{i} \omega+D_{0} q^{2}-\frac{L_{r, 0} R}{\rho_{0} M} F_{\mathrm{m}}
\end{array}\right)(2 \pi)^{3} \delta\left(\mathbf{q}-\mathbf{q}^{\prime}\right) \\
& +i \frac{L_{r, 0} R}{\rho_{0} M} F_{1}\left(\begin{array}{ll}
0 & 0 \\
0 & 1
\end{array}\right)(2 \pi)^{3} \\
& \times \frac{\partial}{\partial q_{x}} \delta\left(q_{x}-q_{x}^{\prime}\right) \delta\left(q_{y}-q_{y}^{\prime}\right) \delta\left(q_{z}-q_{z}^{\prime}\right) \\
\equiv & \mathrm{G}_{0}^{-1}\left(\omega, \mathbf{q}, \mathbf{q}^{\prime}\right)+\mathrm{G}_{1}^{-1}\left(\omega, \mathbf{q}, \mathbf{q}^{\prime}\right) .
\end{aligned}
$$

It follows that

$$
\begin{aligned}
\mathrm{G}_{0}\left(\omega, \mathbf{q}, \mathbf{q}^{\prime}\right)= & \frac{(2 \pi)^{3} \delta\left(\mathbf{q}-\mathbf{q}^{\prime}\right)}{v_{0} q^{4}\left(\mathrm{i} \omega+D_{0} q^{2}-\frac{L_{r, 0} R}{\rho_{0} M} F_{\mathrm{m}}\right)} \\
& \times\left[\begin{array}{cc}
\left(\mathrm{i} \omega+D_{0} q^{2}-\frac{L_{r, 0} R}{\rho_{0} M} F_{\mathrm{m}}\right) & 0 \\
-(\nabla c)_{\mathrm{m}} & \nu_{0} q^{4}
\end{array}\right] \\
\equiv & \mathrm{G}_{0}(\omega, q)(2 \pi)^{3} \delta\left(\mathbf{q}-\mathbf{q}^{\prime}\right) .
\end{aligned}
$$

In order to obtain $G$, we treat $G_{1}^{-1}$ as a perturbation and write

$$
\begin{aligned}
\mathbf{G}\left(\omega, \mathbf{q}, \mathbf{q}^{\prime}\right)= & \mathrm{G}_{0}\left(\omega, \mathbf{q}, \mathbf{q}^{\prime}\right)-(2 \pi)^{-6} \int d \mathbf{q}^{\prime \prime} \\
& \times \int d \mathbf{q}^{\prime \prime \prime} \mathbf{G}_{0}\left(\omega, \mathbf{q}, \mathbf{q}^{\prime \prime}\right) \\
& \cdot \mathbf{G}_{1}^{-1}\left(\omega, \mathbf{q}^{\prime \prime}, \mathbf{q}^{\prime \prime \prime}\right) \cdot \mathbf{G}_{0}\left(\omega, \mathbf{q}^{\prime \prime \prime}, \mathbf{q}^{\prime}\right) \\
= & \mathrm{G}_{0}(\omega, q)(2 \pi)^{3} \delta\left(\mathbf{q}-\mathbf{q}^{\prime}\right) \\
& -\mathbf{G}_{0}(\omega, q) \cdot \mathbf{G}_{1}^{-1}\left(\omega, \mathbf{q}, \mathbf{q}^{\prime}\right) \\
& \cdot \mathrm{G}_{0}\left(\omega, q^{\prime}\right) \\
= & (2 \pi)^{3} \delta\left(q_{y}-q_{y}^{\prime}\right) \delta\left(q_{z}-q_{z}^{\prime}\right)\left[\mathbf{G}_{0}(\omega, q) \delta\left(q_{x}-q_{x}^{\prime}\right)\right. \\
& -i \frac{L_{r, 0} R}{\rho_{0} M} F_{1} \mathbf{G}_{0}(\omega, q) \\
& \left.\cdot\left(\begin{array}{ll}
0 & 0 \\
0 & 1
\end{array}\right) \cdot \mathbf{G}_{0}\left(\omega, q^{\prime}\right) \frac{\partial}{\partial q_{x}} \delta\left(q_{x}-q_{x}^{\prime}\right)\right] \\
\equiv & \mathbf{G}_{0}(\omega, q)(2 \pi)^{3} \delta\left(\mathbf{q}-\mathbf{q}^{\prime}\right)+\mathbf{G}_{1}\left(\omega, q, q^{\prime}\right)(2 \pi)^{3} \\
& \times \delta\left(\mathbf{q}_{\|}-\mathbf{q}_{\|}^{\prime}\right) \frac{\partial}{\partial q_{x}} \delta\left(q_{x}-q_{x}^{\prime}\right) \\
\equiv & \mathbf{G}_{0}\left(\omega, \mathbf{q}, \mathbf{q}^{\prime}\right)+\mathbf{G}_{1}\left(\omega, \mathbf{q}, \mathbf{q}^{\prime}\right) . \\
&
\end{aligned}
$$


After some algebra, this gives

$$
\mathrm{G}_{1}\left(\omega, q, q^{\prime}\right)=\frac{-i \frac{L_{r, 0} R}{\rho_{0} M} F_{1}}{\left[i \omega+D_{0} q^{2}-\frac{L_{r, 0} R}{\rho_{0} M} F_{\mathrm{m}}\right]} \frac{1}{\left[i \omega+D_{0} q^{\prime 2}-\frac{L_{r, 0} R}{\rho_{0} M} F_{\mathrm{m}}\right]}\left[\begin{array}{cc}
0 & 0 \\
-\frac{(\nabla c)_{\mathrm{m}}}{v_{0} q^{\prime 4}} & 1
\end{array}\right] .
$$

The Fourier-transformed fluctuating fields can then be simply evaluated from Eq. (18). We are interested in the autocorrelation function of the (Fourier-transformed) concentration fluctuations, i.e., $\left\langle\delta c^{*}(\omega, \mathbf{q}) \delta c\left(\omega^{\prime}, \mathbf{q}^{\prime}\right)\right\rangle$. For a calculation of this quantity, we need the correlations between the components of the random noise vector introduced in the RHS of Eq. (17).

These functions are conveniently expressed in terms of a correlation matrix $\mathrm{C}\left(t, t^{\prime}, \mathbf{r}, \mathbf{r}^{\prime}\right)$, defined by

$$
\begin{aligned}
\mathrm{C}\left(t, t^{\prime}, \mathbf{r}, \mathbf{r}^{\prime}\right) \equiv & \left\langle F_{i}(t, \mathbf{r}) F_{j}^{\dagger}\left(t^{\prime}, \mathbf{r}^{\prime}\right)\right\rangle=\frac{1}{\rho_{0}^{2}}\left\langle\left(\begin{array}{c}
-\left\{\nabla \times \nabla \times\left(\nabla \cdot \delta \Pi^{(\mathrm{st})}(\mathbf{r}, t)\right)\right\}_{x} \\
-\nabla \cdot \delta \mathbf{J}(\mathbf{r}, t)+\delta r(\mathbf{r}, t)
\end{array}\right)\right. \\
& \left.\times\left(\begin{array}{c}
-\left\{\nabla^{\prime} \times \nabla^{\prime} \times\left(\nabla^{\prime} \cdot \delta \Pi^{(\mathrm{stt})}\left(\mathbf{r}^{\prime}, t^{\prime}\right)\right)\right\}_{x} \\
-\nabla^{\prime} \cdot \delta \mathbf{J}\left(\mathbf{r}^{\prime}, t^{\prime}\right)+\delta r\left(\mathbf{r}^{\prime}, t^{\prime}\right)
\end{array}\right)\right\rangle,
\end{aligned}
$$

where the superscript $\dagger$ indicates a Hermitian conjugate. Using the fluctuation-dissipation theorems given in Eqs. (8), assuming that the Onsager coefficients $(T \eta)_{0}, L_{J J, 0}$ and $L_{r, 0}$ can be taken to be constant and expanding around the location $x_{\mathrm{m}}$, where the diffusion flux is maximum, and also using Eq. (11), we obtain

$$
\begin{aligned}
\mathrm{C}\left(t, t^{\prime}, \mathbf{r}, \mathbf{r}^{\prime}\right)= & \frac{2 k_{\mathrm{B}}}{\rho_{0}^{2}} \delta\left(t-t^{\prime}\right) \delta\left(\mathbf{r}-\mathbf{r}^{\prime}\right)\left(\begin{array}{cc}
(T \eta)_{0} \nabla_{\|} \cdot \nabla_{\|}(\nabla \cdot \nabla)^{2} & 0 \\
0 & L_{J J, 0} \nabla \cdot \nabla+\frac{1}{2} L_{r, 0}\left[1+\exp \left(-\frac{M \Delta g_{0}(x)}{R T_{0}(x)}\right)\right]
\end{array}\right) \\
= & \frac{2 k_{\mathrm{B}}}{\rho_{0}^{2}}\left\{\left(\begin{array}{cc}
(T \eta)_{0} \boldsymbol{\nabla}_{\|} \cdot \nabla_{\|}(\nabla \cdot \nabla)^{2} & 0 \\
0 & L_{J J, 0} \boldsymbol{\nabla} \cdot \nabla+L_{r, 0}
\end{array}\right)\right. \\
& \left.+\frac{1}{2} L_{r, 0}\left[\frac{\partial}{\partial x} \exp \left(-\frac{M \Delta g}{R T}\right)\right]_{\mathrm{m}}\left(\begin{array}{ll}
0 & 0 \\
0 & 1
\end{array}\right)\left(x-x_{\mathrm{m}}\right)\right\} \delta\left(t-t^{\prime}\right) \delta\left(\mathbf{r}-\mathbf{r}^{\prime}\right) .
\end{aligned}
$$

After applying a Fourier transformation and adopting $(T \eta)_{0}=T_{\mathrm{m}} \rho_{0} \nu_{0}$, one obtains

$$
\begin{aligned}
\mathrm{C}\left(\omega, \omega^{\prime}, \mathbf{q}, \mathbf{q}^{\prime}\right)= & \left\langle F_{i}(\omega, \mathbf{q}) F_{j}^{\dagger}\left(\omega^{\prime}, \mathbf{q}^{\prime}\right)\right\rangle=\frac{2 k_{\mathrm{B}}}{\rho_{0}^{2}}\left\{\left(\begin{array}{cc}
T_{\mathrm{m}} \rho_{0} \nu_{0} q_{\|}^{2} q^{4} & 0 \\
0 & L_{J J, 0} q^{2}+L_{r, 0}
\end{array}\right)\right. \\
& \left.-\frac{i}{2} L_{r, 0}\left[\frac{\partial}{\partial x} \exp \left(-\frac{M \Delta g}{R T}\right)\right]_{\mathrm{m}}\left(\begin{array}{cc}
0 & 0 \\
0 & 1
\end{array}\right) \frac{\partial}{\partial q_{x}}\right\}(2 \pi)^{4} \delta\left(\omega-\omega^{\prime}\right) \delta\left(\mathbf{q}-\mathbf{q}^{\prime}\right) \\
\equiv & \mathrm{C}_{0}\left(\omega, \omega^{\prime}, \mathbf{q}, \mathbf{q}^{\prime}\right)+\mathrm{C}_{1}\left(\omega, \omega^{\prime}, \mathbf{q}, \mathbf{q}^{\prime}\right) \\
\equiv & \mathrm{C}_{0}(\mathbf{q})(2 \pi)^{4} \delta\left(\omega-\omega^{\prime}\right) \delta\left(\mathbf{q}-\mathbf{q}^{\prime}\right)+\mathrm{C}_{1}(2 \pi)^{4} \delta\left(\omega-\omega^{\prime}\right) \delta\left(\mathbf{q}_{\|}-\mathbf{q}_{\|}^{\prime}\right) \frac{\partial}{\partial q_{x}} \delta\left(q_{x}-q_{x}^{\prime}\right),
\end{aligned}
$$

where $q_{\|}^{2}=q_{y}^{2}+q_{z}^{2}$, with $q_{\|}$being the component of the wave vector in the direction normal to that of the stationary concentration gradient. Furthermore, the differentiation with respect to $q_{x}$ works on the $\delta$-function outside the curly bracket. We note for future use that

$$
\begin{aligned}
& {\left[\frac{\partial}{\partial x} \exp \left(-\frac{M \Delta g}{R T}\right)\right]_{\mathrm{m}}=-(\nabla \hat{g})_{\mathrm{m}}} \\
& \quad=-\frac{M}{R T_{\mathrm{m}}}\left[\frac{\partial \Delta g}{\partial c} \nabla c-\frac{\Delta h}{T} \nabla T\right]_{\mathrm{m}},
\end{aligned}
$$

where, as in the Appendix, we introduced a dimensionless $\hat{g} \equiv M \Delta g_{0} / R T_{0}$ for ease of notation and used Eq. (11). As a consequence of Eq. (30), the correction $C_{1}$ in Eq. (29) is actually linear in both the concentration and the temperature gradients at the point of maximum stationary flow (i.e., near the middle of the layer).

The validity of the fluctuation-dissipation theorem, as given in Eqs. (28) and (29) for non-equilibrium states far from equilibrium, is a consequence of its validity in the general form given in Eqs. (8). In the previous paper, ${ }^{14}$ we gave a general derivation using mesoscopic non-equilibrium thermodynamics. The physics behind this extension of the fluctuation-dissipation theorem to non-equilibrium states is that the correlation between the components of the stochastic part of the fluxes continues to be short ranged and, thus, within a hydrodynamic theory, proportional to delta functions in space and time. The validity of such an extension of the fluctuation-dissipation theorem has been confirmed experimentally for (non-reacting) fluids in a temperature gradient. $^{7,37,41}$ 


\section{Correlation functions of the fluctuations}

Inverting Eq. (17), we obtain for the fluctuations

$$
\left(\begin{array}{c}
\delta v_{x}(\omega, \mathbf{q}) \\
\delta c(\omega, \mathbf{q})
\end{array}\right)=\left(\frac{1}{2 \pi}\right)^{3} \int d \mathbf{q}^{\prime} \mathbf{G}\left(\omega, \mathbf{q}, \mathbf{q}^{\prime}\right) \cdot \mathbf{F}\left(\omega, \mathbf{q}^{\prime}\right),
$$

and, hence, for the correlations of the fluctuations,

$$
\begin{aligned}
\mathrm{S}\left(\omega, \omega^{\prime}, \mathbf{q}, \mathbf{q}^{\prime}\right) \equiv & \left\langle\left(\begin{array}{c}
\delta v_{x}(\omega, \mathbf{q}) \\
\delta c(\omega, \mathbf{q})
\end{array}\right)\left(\begin{array}{c}
\delta v_{x}\left(\omega^{\prime}, \mathbf{q}^{\prime}\right) \\
\delta c\left(\omega^{\prime}, \mathbf{q}^{\prime}\right)
\end{array}\right)^{\dagger}\right\rangle \\
= & \left(\frac{1}{2 \pi}\right)^{6} \int d \mathbf{q}^{\prime \prime} d \mathbf{q}^{\prime \prime \prime} \mathbf{G}\left(\omega, \mathbf{q}, \mathbf{q}^{\prime \prime}\right) \cdot \mathbf{C}\left(\omega, \omega^{\prime}, \mathbf{q}^{\prime \prime}, \mathbf{q}^{\prime \prime \prime}\right) \cdot \mathbf{G}^{\dagger}\left(\omega^{\prime}, \mathbf{q}^{\prime \prime \prime}, \mathbf{q}^{\prime}\right) \\
= & \left(\frac{1}{2 \pi}\right)^{6} \int d \mathbf{q}^{\prime \prime} d \mathbf{q}^{\prime \prime \prime} \mathbf{G}_{0}\left(\omega, \mathbf{q}, \mathbf{q}^{\prime \prime}\right) \cdot \mathbf{C}_{0}\left(\omega, \omega^{\prime}, \mathbf{q}^{\prime \prime}, \mathbf{q}^{\prime \prime \prime}\right) \cdot \mathbf{G}_{0}^{\dagger}\left(\omega^{\prime}, \mathbf{q}^{\prime \prime \prime}, \mathbf{q}^{\prime}\right) \\
& +\left(\frac{1}{2 \pi}\right)^{6} \int d \mathbf{q}^{\prime \prime} d \mathbf{q}^{\prime \prime \prime} \mathbf{G}_{1}\left(\omega, \mathbf{q}, \mathbf{q}^{\prime \prime}\right) \cdot \mathbf{C}_{0}\left(\omega, \omega^{\prime}, \mathbf{q}^{\prime \prime}, \mathbf{q}^{\prime \prime \prime}\right) \cdot \mathbf{G}_{0}^{\dagger}\left(\omega^{\prime}, \mathbf{q}^{\prime \prime \prime}, \mathbf{q}^{\prime}\right) \\
& +\left(\frac{1}{2 \pi}\right)^{6} \int d \mathbf{q}^{\prime \prime} d \mathbf{q}^{\prime \prime \prime} \mathbf{G}_{0}\left(\omega, \mathbf{q}, \mathbf{q}^{\prime \prime}\right) \cdot \mathrm{C}_{1}\left(\omega, \omega^{\prime}, \mathbf{q}^{\prime \prime}, \mathbf{q}^{\prime \prime \prime}\right) \cdot \mathbf{G}_{0}^{\dagger}\left(\omega^{\prime}, \mathbf{q}^{\prime \prime \prime}, \mathbf{q}^{\prime}\right) \\
& +\left(\frac{1}{2 \pi}\right)^{6} \int d \mathbf{q}^{\prime \prime} d \mathbf{q}^{\prime \prime \prime} \mathbf{G}_{0}\left(\omega, \mathbf{q}, \mathbf{q}^{\prime \prime}\right) \cdot \mathbf{C}_{0}\left(\omega, \omega^{\prime}, \mathbf{q}^{\prime \prime}, \mathbf{q}^{\prime \prime \prime}\right) \cdot \mathbf{G}_{1}^{\dagger}\left(\omega^{\prime}, \mathbf{q}^{\prime \prime \prime}, \mathbf{q}^{\prime}\right) \\
\equiv & \mathrm{S}_{0}\left(\omega, \omega^{\prime}, \mathbf{q}, \mathbf{q}^{\prime}\right)+\mathrm{S}_{1}\left(\omega, \omega^{\prime}, \mathbf{q}, \mathbf{q}^{\prime}\right)+\mathrm{S}_{2}\left(\omega, \omega^{\prime}, \mathbf{q}, \mathbf{q}^{\prime}\right)+\mathrm{S}_{1}^{\dagger}\left(\omega, \omega^{\prime}, \mathbf{q}, \mathbf{q}^{\prime}\right) .
\end{aligned}
$$

In this expression, we expanded to the first order in the correction term due to the gradient which is important far from equilibrium. Both $S_{0}$ and $S_{2}$ are Hermitian, while $S_{1}+S_{1}^{\dagger}$ is also Hermitian. Substituting the expressions obtained for $G$ and $C$, given in Eqs. (24)-(26) and (29), into Eq. (32) we find

$$
\begin{aligned}
& \mathrm{S}_{0}\left(\omega, \omega^{\prime}, \mathbf{q}, \mathbf{q}^{\prime}\right)=\mathbf{G}_{0}(\omega, q) \cdot \mathrm{C}_{0}(\mathbf{q}) \cdot \mathbf{G}_{0}^{\dagger}(\omega, q)(2 \pi)^{4} \delta\left(\omega-\omega^{\prime}\right) \delta\left(\mathbf{q}-\mathbf{q}^{\prime}\right) \\
& =\frac{2 k_{\mathrm{B}}}{\rho_{0}^{2}}(2 \pi)^{4} \delta\left(\omega-\omega^{\prime}\right) \delta\left(\mathbf{q}-\mathbf{q}^{\prime}\right) \\
& \times \frac{1}{v_{0} q^{4}\left(\mathrm{i} \omega+D_{0} q^{2}-\frac{L_{r, 0} R}{\rho_{0} M} F_{\mathrm{m}}\right)}\left[\begin{array}{cc}
\left(\mathrm{i} \omega+D_{0} q^{2}-\frac{L_{r, 0} R}{\rho_{0} M} F_{\mathrm{m}}\right) & 0 \\
-(\nabla c)_{\mathrm{m}} & \nu_{0} q^{4}
\end{array}\right] \\
& \cdot\left[\begin{array}{cc}
T_{\mathrm{m}} \rho_{0} v_{0} q_{\|}^{2} q^{4} & 0 \\
0 & L_{J J, 0} q^{2}+L_{r, 0}
\end{array}\right] \\
& \cdot \frac{1}{\nu_{0} q^{4}\left(-\mathrm{i} \omega+D_{0} q^{2}-\frac{L_{r, 0} R}{\rho_{0} M} F_{\mathrm{m}}\right)}\left[\begin{array}{cc}
\left(-\mathrm{i} \omega+D_{0} q^{2}-\frac{L_{r, 0} R}{\rho_{0} M} F_{\mathrm{m}}\right) & -(\nabla c)_{\mathrm{m}} \\
0 & \nu_{0} q^{4}
\end{array}\right], \\
& \mathrm{S}_{1}\left(\omega, \omega^{\prime}, \mathbf{q}, \mathbf{q}^{\prime}\right)=\mathrm{G}_{1}\left(\omega, q, q^{\prime}\right) \cdot \mathrm{C}_{0}\left(\omega, \mathbf{q}^{\prime}\right) \cdot \mathbf{G}_{0}^{\dagger}\left(\omega, q^{\prime}\right)(2 \pi)^{4} \delta\left(\omega-\omega^{\prime}\right) \delta\left(\mathbf{q}_{\|}-\mathbf{q}_{\|}^{\prime}\right) \frac{\partial}{\partial q_{x}} \delta\left(q_{x}-q_{x}^{\prime}\right) \\
& =-i \frac{2 k_{\mathrm{B}}}{\rho_{0}^{3}} \frac{L_{r, 0} R}{M} F_{1}(2 \pi)^{4} \delta\left(\omega-\omega^{\prime}\right) \delta\left(\mathbf{q}_{\|}-\mathbf{q}_{\|}^{\prime}\right) \frac{\partial}{\partial q_{x}} \delta\left(q_{x}-q_{x}^{\prime}\right)\left(i \omega+D_{0} q^{2}-\frac{L_{r, 0} R}{\rho_{0} M} F_{\mathrm{m}}\right)^{-1} \\
& \times\left(i \omega+D_{0} q^{\prime 2}-\frac{L_{r, 0} R}{\rho_{0} M} F_{\mathrm{m}}\right)^{-1} \times\left(\begin{array}{cc}
0 & 0 \\
-(\nabla c)_{\mathrm{m}}\left(\nu_{0} q^{\prime 4}\right)^{-1} & 1
\end{array}\right) \\
& \cdot\left[\begin{array}{cc}
T_{\mathrm{m}} \rho_{0} v_{0} q_{\|}^{\prime 2} q^{\prime 4} & 0 \\
0 & L_{J J, 0} q^{\prime 2}+L_{r, 0}
\end{array}\right] \\
& \cdot \frac{1}{v_{0} q^{\prime 4}\left(-\mathrm{i} \omega+D_{0} q^{\prime 2}-\frac{L_{r, 0} R}{\rho_{0} M} F_{\mathrm{m}}\right)}\left[\begin{array}{cc}
\left(-\mathrm{i} \omega+D_{0} q^{\prime 2}-\frac{L_{r, 0} R}{\rho_{0} M} F_{\mathrm{m}}\right) & -(\nabla c)_{\mathrm{m}} \\
0 & \nu_{0} q^{\prime 4}
\end{array}\right] \text {, }
\end{aligned}
$$




$$
\begin{aligned}
\mathrm{S}_{2}\left(\omega, \omega^{\prime}, \mathbf{q}, \mathbf{q}^{\prime}\right)= & \mathrm{G}_{0}(\omega, q) \cdot \mathrm{C}_{1} \cdot \mathrm{G}_{0}^{\dagger}\left(\omega, q^{\prime}\right)(2 \pi)^{4} \delta\left(\omega-\omega^{\prime}\right) \delta\left(\mathbf{q}_{\|}-\mathbf{q}_{\|}^{\prime}\right) \frac{\partial}{\partial q_{x}} \delta\left(q_{x}-q_{x}^{\prime}\right) \\
= & -i \frac{k_{\mathrm{B}}}{\rho_{0}^{2}} L_{r, 0}\left[\frac{\partial}{\partial x} \exp \left(-\frac{M \Delta g}{R T}\right)\right]_{\mathrm{m}}(2 \pi)^{4} \delta\left(\omega-\omega^{\prime}\right) \delta\left(\mathbf{q}_{\|}-\mathbf{q}_{\|}^{\prime}\right) \frac{\partial}{\partial q_{x}} \delta\left(q_{x}-q_{x}^{\prime}\right) \\
& \times\left(\nu_{0} q^{4}\right)^{-1}\left(i \omega+D_{0} q^{2}-\frac{L_{r, 0} R}{\rho_{0} M} F_{\mathrm{m}}\right)^{-1} \times\left[\begin{array}{cc}
\left(\mathrm{i} \omega+D_{0} q^{2}-\frac{L_{r, 0} R}{\rho_{0} M} F_{\mathrm{m}}\right) & 0 \\
-(\nabla c)_{\mathrm{m}} & \nu_{0} q^{4}
\end{array}\right] \cdot\left(\begin{array}{cc}
0 & 0 \\
0 & 1
\end{array}\right) \\
& \cdot\left(v_{0} q^{\prime 4}\right)^{-1}\left(-i \omega+D_{0} q^{\prime 2}-\frac{L_{r, 0} R}{\rho_{0} M} F_{\mathrm{m}}\right)^{-1}\left[\begin{array}{cc}
\left(-\mathrm{i} \omega+D_{0} q^{\prime 2}-\frac{L_{r, 0} R}{\rho_{0} M} F_{\mathrm{m}}\right) & -(\nabla c)_{\mathrm{m}} \\
0 & \nu_{0} q^{\prime 4}
\end{array}\right] \\
= & -i \frac{k_{\mathrm{B}}}{\rho_{0}^{2}} L_{r, 0}\left[\frac{\partial}{\partial x} \exp \left(-\frac{M \Delta g}{R T}\right)\right]_{\mathrm{m}}(2 \pi)^{4} \delta\left(\omega-\omega^{\prime}\right) \delta\left(\mathbf{q}_{\|}-\mathbf{q}_{\|}^{\prime}\right) \frac{\partial}{\partial q_{x}} \delta\left(q_{x}-q_{x}^{\prime}\right) \\
& \times\left(i \omega+D_{0} q^{2}-\frac{L_{r, 0} R}{\rho_{0} M} F_{\mathrm{m}}\right)^{-1}\left(-i \omega+D_{0} q^{\prime 2}-\frac{L_{r, 0} R}{\rho_{0} M} F_{\mathrm{m}}\right)^{-1}\left(\begin{array}{ll}
0 & 0 \\
0 & 1
\end{array}\right),
\end{aligned}
$$

where the derivative of the delta function works on the delta function alone. The Hermitian conjugate of $S_{1}$ is obtained by interchanging the matrices taking the mirror images of the first and the last, taking the complex conjugate, and interchanging $\omega$ and $\omega^{\prime}, q$ and $q^{\prime}, q_{x}$ and $q_{x}^{\prime}$. As we will not need the explicit form of this operator, we do not give it.

For the zeroth-order concentration correlation function, Eq. (33) gives

$$
S_{c c, 0}\left(\omega, \omega^{\prime}, \mathbf{q}, \mathbf{q}^{\prime}\right)=S_{c c, 0}(\omega, q)(2 \pi)^{4} \delta\left(\omega-\omega^{\prime}\right) \delta\left(\mathbf{q}-\mathbf{q}^{\prime}\right)
$$

with

$$
S_{c c, 0}(\omega, q)=\frac{2 k_{\mathrm{B}}}{\rho_{0}^{2}} \frac{L_{J J, 0} q^{2}+L_{r, 0}+(\nabla c)_{\mathrm{m}}^{2} T_{\mathrm{m}} \rho_{0} v_{0}^{-1} q_{\|}^{2} q^{-4}}{\left[\omega^{2}+\left(D_{0} q^{2}-\frac{L_{r, 0} R}{\rho_{0} M} F_{\mathrm{m}}\right)^{2}\right]} .
$$

This results in

$$
S_{c c, 0}(\omega, q)=\frac{2 k_{\mathrm{B}} T_{\mathrm{m}}}{\rho_{0}} \frac{D_{0}\left(\frac{\partial \Delta g}{\partial c}\right)_{T, \mathrm{~m}}^{-1} q^{2}+\frac{L_{r, 0}}{\rho_{0} T_{\mathrm{m}}}+\frac{q_{\|}^{2}}{v_{0} q^{4}}(\nabla c)_{\mathrm{m}}^{2}}{\omega^{2}+\left[D_{0} q^{2}+\frac{L_{r, 0}}{\rho_{0} T_{\mathrm{m}}}\left(\frac{\partial \Delta g}{\partial c}\right)_{T, \mathrm{~m}}\right]^{2}}
$$

where $L_{J J, 0}$ was substituted from Eq. (5), $F_{\mathrm{m}}$ from Eq. (20a), and Eq. (11) has been used to simplify the resulting expression.

From Eqs. (34) and (35) and also using Eq. (20) we obtain for the first-order corrections to the concentration correlation function,

$$
S_{c c, 1}\left(\omega, \omega^{\prime}, \mathbf{q}, \mathbf{q}^{\prime}\right)=S_{c c, 1}\left(\omega, \mathbf{q}_{\|}, q_{x}, q_{x}^{\prime}\right)(2 \pi)^{4} \delta\left(\omega-\omega^{\prime}\right) \delta\left(\mathbf{q}_{\|}-\mathbf{q}_{\|}^{\prime}\right) \frac{\partial}{\partial q_{x}} \delta\left(q_{x}-q_{x}^{\prime}\right)
$$

with

$$
S_{c c, 1}\left(\omega, \mathbf{q}_{\|}, q_{x}, q_{x}^{\prime}\right)=\frac{-2 i \frac{k_{B} L_{r, 0} R T_{\mathrm{m}} F_{1}}{\rho_{0}^{2} M}\left[D_{0}\left(\frac{\partial \Delta g}{\partial c}\right)_{T, \mathrm{~m}}^{-1} q^{\prime 2}+\frac{L_{r, 0}}{\rho_{0} T_{\mathrm{m}}}+\frac{q_{\|}^{\prime 2}(\nabla c)_{\mathrm{m}}^{2}}{\nu_{0} q^{\prime 4}}\right]}{\left(i \omega+D_{0} q^{2}+\frac{L_{r, 0}}{\rho_{0} T_{\mathrm{m}}}\left(\frac{\partial \Delta g}{\partial c}\right)_{T, \mathrm{~m}}\right)\left[\omega^{2}+\left(D_{0} q^{\prime 2}+\frac{L_{r, 0}}{\rho_{0} T_{\mathrm{m}}}\left(\frac{\partial \Delta g}{\partial c}\right)_{T, \mathrm{~m}}\right)^{2}\right]},
$$

and, similarly, using also Eq. (30),

$$
S_{c c, 2}\left(\omega, \omega^{\prime}, \mathbf{q}, \mathbf{q}^{\prime}\right)=S_{c c, 2}\left(\omega, \mathbf{q}_{\|}, q_{x}, q_{x}^{\prime}\right)(2 \pi)^{4} \delta\left(\omega-\omega^{\prime}\right) \delta\left(\mathbf{q}_{\|}-\mathbf{q}_{\|}^{\prime}\right) \frac{\partial}{\partial q_{x}} \delta\left(q_{x}-q_{x}^{\prime}\right)
$$

with

$$
S_{c c, 2}\left(\omega, \mathbf{q}_{\|}, q_{x}, q_{x}^{\prime}\right)=\frac{i \frac{k_{\mathrm{B}} M L_{r, 0}}{R T_{\mathrm{m}} \rho_{0}^{2}}\left[\left(\frac{\partial \Delta g}{\partial c}\right)_{T} \nabla c-\frac{\Delta h}{T} \nabla T\right]_{\mathrm{m}}}{\left[-i \omega+D_{0} q^{2}+\frac{L_{r, 0}}{\rho_{0} T_{\mathrm{m}}}\left(\frac{\partial \Delta g}{\partial c}\right)_{T, \mathrm{~m}}\right]\left[i \omega+D_{0} q^{\prime 2}+\frac{L_{r, 0}}{\rho_{0} T_{\mathrm{m}}}\left(\frac{\partial \Delta g}{\partial c}\right)_{T, \mathrm{~m}}\right]} .
$$

Note that the derivative of the $\delta$-function works only on the $\delta$-function. Hence, the two first-order corrections, $S_{c c, 1}$ and $S_{c c, 2}$, are linear in the stationary gradients of both the concentration and the temperature at the location where the diffusion flow has a maximum. 


\section{PHYSICAL INTERPRETATION OF THE NON-EQUILIBRIUM CONCENTRATION CORRELATION FUNCTIONS}

To explain the physical meaning of the equations derived in Sec. IV for the non-equilibrium concentration correlation functions, we first elucidate in Subsection V A the intensity and the wave-number dependence of the non-equilibrium concentration fluctuations implied by the zeroth-order approximation, Eq. (37). In Subsection V B, we discuss the first-order corrections to the concentration fluctuations due to spatial inhomogeneities. Our conclusions are summarized in Sec. VI.

\section{A. Non-equilibrium enhancement due to mode coupling}

We observe from Eq. (37) that, in a zeroth-order approximation, the autocorrelation of concentration fluctuations calculated on the basis of the Kramers kinetics (2) is essentially the same as the one obtained previously ${ }^{13}$ on the basis of linear kinetics (1), the latter analyzed in the previous publication. ${ }^{13}$ The only significant difference is that in the case of linear kinetics (1) all the thermophysical properties appearing in Eq. (37) are to be taken at their values at the midpoint $(x=L / 2)$, while in the case of Kramers kinetics (1) these values are to be taken at the point of maximum stationary diffusion flux $\left(x=x_{\mathrm{m}}\right)$, which no longer coincides with the midpoint.

It is illustrative to rewrite Eq. (37) as

$$
\begin{aligned}
& S_{c c, 0}(\omega, q) \\
& \quad=S_{c c}^{(\mathrm{E})}(\omega, q)\left\{1+\frac{q_{\|}^{2}\left(\frac{\partial \Delta g}{\partial c}\right)_{T, \mathrm{~m}}(\nabla c)_{\mathrm{m}}^{2}}{v_{0} q^{4}\left[D_{0} q^{2}+\frac{L_{r, 0}}{\rho_{0} T_{\mathrm{m}}}\left(\frac{\partial \Delta g}{\partial c}\right)_{T, \mathrm{~m}}\right]}\right\},
\end{aligned}
$$

where

$$
\begin{aligned}
& S_{c c}^{(\mathrm{E})}(\omega, q) \\
& =\frac{k_{\mathrm{B}} T_{\mathrm{m}}}{\rho_{0}}\left(\frac{\partial \Delta g}{\partial c}\right)_{T, \mathrm{~m}}^{-1} \frac{2\left[D_{0} q^{2}+\frac{L_{r, 0}}{\rho_{0} T_{\mathrm{m}}}\left(\frac{\partial \Delta g}{\partial c}\right)_{T, \mathrm{~m}}\right]}{\omega^{2}+\left[D_{0} q^{2}+\frac{L_{r, 0}}{\rho_{0} T_{\mathrm{m}}}\left(\frac{\partial \Delta g}{\partial c}\right)_{T, \mathrm{~m}}\right]^{2}}
\end{aligned}
$$

is the spectrum of fluctuations if the system were in equilibrium at the temperature $T_{\mathrm{m}}$ corresponding to the location of maximum diffusion flow. As discussed in Ref. 24 (see also Sec. IV A), this temperature differs from the harmonic average of the boundary temperatures in the case of Kramers kinetics (2), while in the case of linear kinetics (1) this temperature equals the harmonic average of the boundary temperatures.

An interesting feature that can be inferred from Eqs. (42) and (43) is that the decay rate of non-equilibrium concentration fluctuations is the same as the decay rate of equilibrium concentration fluctuations, which in the same
large-Lewis-number approximation used here was discussed in our first paper. ${ }^{13}$ This is a generic feature of nonequilibrium fluctuations, namely, that for sufficiently large wave numbers the decay rate of the fluctuations is the same, while the intensity of the fluctuations is strongly affected by the non-equilibrium constraints, ${ }^{7}$ as obviously shown in Eq. (42). However, inclusion of the effects of buoyancy and of boundary conditions does modify the decay rate of nonequilibrium fluctuations. ${ }^{7}$

The static structure factor $S_{c c, 0}(q)$, which represents the total intensity of the non-equilibrium concentration fluctuations in zeroth approximation, is obtained by integrating $S_{c c, 0}(\omega, q)$ over the frequency $\omega$. In terms of a dimensionless wave number $\tilde{q}=q L$,

$$
\begin{aligned}
S_{c c, 0}(\mathbf{q}) & =S_{c c}^{(\mathrm{E})}\left\{1+\tilde{S}_{c c}^{(\mathrm{NE})} \frac{\tilde{q}_{\|}^{2}}{\tilde{q}^{4}\left[\tilde{q}^{2}+\frac{L^{2}}{d^{2}}\right]}\right\} \\
& =S_{c c}^{(\mathrm{E})}\left\{1+\tilde{S}_{c c}^{(\mathrm{NE})} \frac{\tilde{q}_{\|}^{2}}{\tilde{q}^{4}\left[\tilde{q}^{2}+\phi^{2}\right]}\right\},
\end{aligned}
$$

where, as in Figs. 1 and 2, $d$ is the penetration depth of the chemical reaction defined by Eq. (A3) in the Appendix, and $\phi=L / d$. The coefficient $S_{c c}^{(\mathrm{E})}$ represents the equilibrium intensity of the concentration fluctuations,

$$
S_{c c}^{(\mathrm{E})}=\frac{k_{\mathrm{B}} T_{\mathrm{m}}}{\rho_{0}}\left(\frac{\partial \Delta g}{\partial c}\right)_{T, \mathrm{~m}}^{-1},
$$

that is unaffected by the chemical kinetics. That the intensity of concentration fluctuations in a reacting system in equilibrium is independent of the chemical reaction kinetics is very well known, as reviewed, for instance, by Berne and Pecora. ${ }^{42}$

The factor $\tilde{S}_{c c}^{(\mathrm{NE})}$ in Eq. (44) is a normalized nonequilibrium enhancement of concentration fluctuations due to mode coupling, and it is given by ${ }^{43}$

$$
\tilde{S}_{c c}^{(\mathrm{NE})}=\frac{(\nabla c)_{\mathrm{m}}^{2} L^{4}}{\nu_{0} D_{0}}\left(\frac{\partial \Delta g}{\partial c}\right)_{T, \mathrm{~m}},
$$

which is always positive and, hence, represents a true nonequilibrium enhancement. We note that the mode-coupling mechanism causing the enhancement of non-equilibrium fluctuations is the term $\delta v_{x} \nabla c_{0}$ in the original working Eq. (15b). Physically this corresponds to the fact that velocity fluctuations parallel to the gradient are mixing regions with different local concentrations. Our final result for the zeroth-order approximation, Eqs. (37) and (44), exhibits the typical structure of non-equilibrium fluctuations, containing a non-equilibrium enhancement which explicitly depends on the wave number $\mathbf{q}$, implying that the equal-time non-equilibrium concentration fluctuations become spatially long ranged.

We find that the non-equilibrium enhancement exhibits a crossover from the well-known $q^{-4}$ dependence observed in non-reacting liquid mixtures ${ }^{7}$ to a $q^{-2}$ dependence for smaller wave numbers. The $q^{-2}$ behavior is the one typical for long-range non-equilibrium fluctuations in isothermal reacting mixtures, as has been discussed by several authors. ${ }^{7,21,44}$ The crossover from a $q^{-4}$ (non-isothermal non-reacting) to a $q^{-2}$ (non-equilibrium but isothermally reacting) behavior 


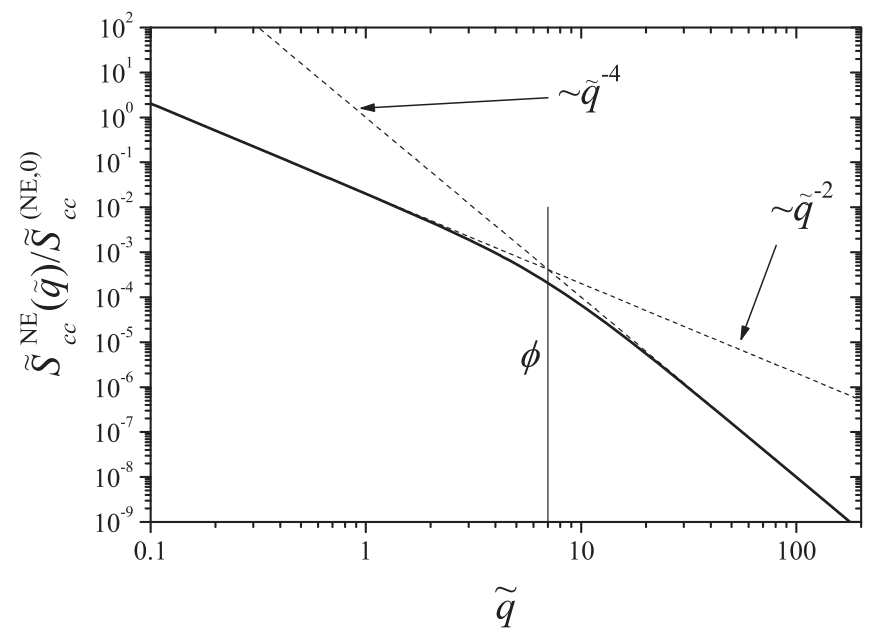

FIG. 3. Zeroth-order enhancement of non-equilibrium concentration fluctuations - see Eq. (44) - as a function of dimensionless wave number, $\tilde{q}=q L$, for parameter $\phi=7$ (fast chemical reaction). The crossover from the asymptotic $q^{-4}$ dependence to a $q^{-2}$ dependence at smaller wave numbers is evident.

occurs at wave numbers of the order,

$$
\tilde{q}_{\mathrm{CO}}^{2} \equiv L^{2} q_{\mathrm{CO}}^{2} \simeq \frac{L^{2}}{d^{2}}=\phi^{2}
$$

which is, therefore, closely related to the inverse of the penetration length $d$ of the stationary solution as given by Eq. (A3). As an illustration, we present in Fig. 3 a plot of the dimensionless non-equilibrium enhancement of the concentration fluctuations as a function of the dimensionless wave number. The plot is for $\phi=7$, which we consider a reasonable value for diffusion-controlled processes (fast chemical reaction). Figure 3 shows a clear crossover from the asymptotic $q^{-4}$ dependence at larger wave numbers (unaffected by the chemical reaction) to a $q^{-2}$ dependence for smaller wave numbers.

\section{B. Non-equilibrium enhancement due to inhomogeneities}

The first-order corrections to the intensity of nonequilibrium fluctuations were presented in Eqs. (38)-(41). We note that these corrections arise from spatial inhomogeneities in the problem. The correction $S_{c c, 1}$ of Eqs. (38) and (39) and its Hermitian conjugate comes from the presence of a spatially non-uniform reaction rate in Eq. (21). The correction $S_{c c, 2}$ of Eqs. (40) and (41) comes from having considered spatially dependent thermal noise in Eq. (28). Both cases have in common that the Fourier-transformed correction to the autocorrelation function is proportional to the derivative of a delta function $\partial_{q_{x}} \delta\left(q_{x}-q_{x}^{\prime}\right)$, and not to a delta function itself, as was the case for the zeroth-order approximation $S_{c c, 0}$. In addition, both corrections are linear in the gradients $(\nabla c)_{\mathrm{m}}$ and $(\nabla T)_{\mathrm{m}}$ as follows from Eqs. (39) and (41).

Expressions containing derivatives of delta functions, such as Eqs. (38) and (40), are commonly encountered when studying the effects of inhomogeneities on the autocorrelation functions of fluctuations. An example is the effect of a thermal gradient in the Brillouin lines of a simple fluid ${ }^{31}$ when boundary conditions are not considered. As discussed in the original papers ${ }^{31}$ and reviewed more in detail in Ref. 7, when the autocorrelation function has the structure (39)-(41), the resulting contributions to the structure factor that would be observed experimentally are given by the diagonal elements of $S_{c c, 1}+S_{c c, 1}^{\dagger}$ and $S_{c c, 2}$. As diagonal elements of Hermitian operators are real, these diagonal elements are given by

$$
\begin{gathered}
S_{c c, 1}(\omega, \mathbf{q})+S_{c c, 1}^{\dagger}(\omega, \mathbf{q}) \\
=-2 \mathcal{R} e\left[\frac{\partial S_{c c, 1}\left(\omega, \mathbf{q}_{\|}, q_{x}, q_{x}^{\prime}\right)}{\partial q_{x}}\right]_{q_{x}^{\prime}=q_{x}}, \\
S_{c c, 2}(\omega, \mathbf{q})=-\mathcal{R} e\left[\frac{\partial S_{c c, 2}\left(\omega, \mathbf{q}_{\|}, q_{x}, q_{x}^{\prime}\right)}{\partial q_{x}}\right]_{q_{x}^{\prime}=q_{x}}
\end{gathered}
$$

with $S_{c c, 1}\left(\omega, \mathbf{q}_{\|}, q_{x}, q_{x}^{\prime}\right)$ and $S_{c c, 2}\left(\omega, \mathbf{q}_{\|}, q_{x}, q_{x}^{\prime}\right)$ given by Eqs. (39) and (41), respectively. Next, from Eqs. (39) and (41), we obtain for the diagonal elements,

$$
\begin{aligned}
& S_{c c, 1}(\omega, \mathbf{q})+S_{c c, 1}^{\dagger}(\omega, \mathbf{q}) \\
& =16 D_{0} \omega q_{x} \frac{k_{\mathrm{B}} T_{\mathrm{m}} F_{1} L_{r, 0}}{\rho_{0}^{2}} \\
& \times\left[D_{0} q^{2}+\frac{L_{r, 0}}{\rho_{0} T_{\mathrm{m}}}\left(\frac{\partial \Delta g}{\partial c}\right)_{T, \mathrm{~m}}\right] \\
& \times \frac{D_{0}\left(\frac{\partial \Delta g}{\partial c}\right)_{T, \mathrm{~m}}^{-1} q^{2}+\frac{L_{r, 0}}{\rho_{0} T_{\mathrm{m}}}+\frac{q_{\|}^{2}}{v_{0} q^{4}}(\nabla c)_{\mathrm{m}}^{2}}{\left[\omega^{2}+\left(D_{0} q^{2}+\frac{L_{r, 0}}{\rho_{0} T_{\mathrm{m}}}\left(\frac{\partial \Delta g}{\partial c}\right)_{T, \mathrm{~m}}\right)^{2}\right]^{2}}, \\
& S_{c c, 2}(\omega, q)=-2 D_{0} \omega q_{x} \frac{L_{r, 0} M}{\rho_{0}^{2} N_{\mathrm{av}} T_{\mathrm{m}}} \frac{\left[\left(\frac{\partial \Delta g}{\partial c}\right)_{T} \nabla c-\frac{\Delta h}{T} \nabla T\right]_{\mathrm{m}}}{{ }} \\
& \times\left[\omega^{2}+\left(D_{0} q^{2}+\frac{L_{r, 0}}{\rho_{0} T_{\mathrm{m}}}\left(\frac{\partial \Delta g}{\partial c}\right)_{T, \mathrm{~m}}\right)^{2}\right]^{2} .
\end{aligned}
$$

Note that, upon integration over $\omega$ of Eq. (49) or Eq. (50), the first-order corrections cancel out. Hence, in first order there is a correction in the dynamic structure factor, but not in the static structure factor. This situation is similar to what happens to the Brillouin lines in the presence of a temperature gradient. $^{31}$

It is interesting to compare the magnitude of the enhancement of non-equilibrium fluctuations due to different sources. Equation (42) contains the enhancement of fluctuations due to mode coupling. In the two new first-order contributions, Eq. (49) contains the enhancement of fluctuations due to an inhomogeneous reaction rate, while Eq. (50) gives the enhancement of fluctuations due to inhomogeneous noise. We first observe that enhancement due to mode coupling in Eq. (42) is quadratic in the gradient $(\nabla c)_{\mathrm{m}}$, while the enhancement due to the first-order contributions are both linear. This fact may lead one to think that the former effect is more important, but caution is required here because of the 
other thermophysical properties involved, as discussed below. Both enhancements are anisotropic, but of different character. The mode-coupling effect in Eq. (42) is maximum for fluctuations with wave vector in the plane parallel to the walls $\left(q_{x} \simeq 0\right)$ and zero for fluctuations with wave vector normal to the walls. On the other hand, the noise inhomogeneity effect is maximum for fluctuations with wave vector normal to the walls $q_{\|} \simeq 0$ and zero for fluctuations with wave vector parallel to the walls. This situation is similar to what happens when comparing the different non-equilibrium enhancements in heat conduction, and we refer to a publication by some of $\mathrm{us}^{32}$ where the physical significance of these features is discussed.

Of maximum interest is to compare the relative strength of non-equilibrium enhancements due to mode coupling and due to noise inhomogeneity. For such a comparison, one has to integrate the corresponding expressions over the frequency $\omega$. One difficulty is that such an integration gives zero for the noise inhomogeneity effect, Eq. (49). We propose to integrate $\omega$ over the half-range $[0, \infty)$ and compare with half the nonequilibrium enhancement due to mode-coupling discussed in Sec. V A . Such an integration yields

$$
\begin{gathered}
S_{c c, 1}^{(1 / 2)}(\mathbf{q})+S_{c c, 1}^{\dagger(1 / 2)}(\mathbf{q})=4 D_{0} q_{x} \frac{k_{\mathrm{B}} L_{r, 0} R T_{\mathrm{m}} F_{1}}{\rho_{0}^{2} M} \\
\times\left[\frac{D_{0}\left(\frac{\partial \Delta g}{\partial c}\right)_{T}^{-1} q^{2}+\frac{L_{r, 0}}{\rho_{0} T}+\frac{q_{\|}^{2}}{\nu_{0} q^{\prime 4}}(\nabla c)^{2}}{D_{0} q^{2}+\frac{L_{r, 0}}{\rho_{0} T}\left(\frac{\partial \Delta g}{\partial c}\right)_{T}}\right]_{\mathrm{m}} \\
S_{c c, 2}^{(1 / 2)}(\mathbf{q})=-D_{0} q_{x} \frac{k_{\mathrm{B}} L_{r, 0} M}{\rho_{0}^{2} R T_{\mathrm{m}}} \frac{\left[\left(\frac{\partial \Delta g}{\partial c}\right)_{T} \nabla c-\frac{\Delta h}{T} \nabla T\right]_{\mathrm{m}}}{\left[D_{0} q^{2}+\frac{L_{r, 0}}{\rho_{0} T_{\mathrm{m}}}\left(\frac{\partial \Delta g}{\partial c}\right)_{T, \mathrm{~m}}\right]^{2}} .
\end{gathered}
$$

Expressing these results in terms of the same dimensionless parameters used in the discussion of the (zeroth-order) correction due to mode coupling, we obtain

$$
\begin{aligned}
& S_{c c, 1}^{(1 / 2)}(\mathbf{q})+S_{c c, 1}^{\dagger(1 / 2)}(\mathbf{q}) \\
& \left.=S_{c c}^{(\mathrm{E})} \phi^{2} \frac{L R T_{\mathrm{m}} F_{1}}{M}\left(\frac{\partial \Delta g}{\partial c}\right)_{T, \mathrm{~m}}^{-1} \frac{4 \tilde{q}_{x}\left[1+\frac{\tilde{S}_{c c}^{(\mathrm{NE})}}{\tilde{q}^{2}+\phi^{2}} \tilde{q}_{\|}^{2}\right.}{\tilde{q}^{4}}\right] \\
& \left(\tilde{q}^{2}+\phi^{2}\right)^{2}
\end{aligned}
$$

$$
\begin{aligned}
S_{c c, 2}^{(1 / 2)}(q)= & -S_{c c}^{(\mathrm{E})} \frac{M L_{r, 0}}{R T_{\mathrm{m}}^{2}}\left(\frac{\partial \Delta g}{\partial c}\right)_{T, \mathrm{~m}} \frac{L^{3}}{\rho_{0} D_{0}} \\
& \times\left[\left(\frac{\partial \Delta g}{\partial c}\right)_{T} \nabla c-\frac{\Delta h}{T} \nabla T\right]_{\mathrm{m}} \frac{\tilde{q}_{x}}{\left(\tilde{q}^{2}+\phi^{2}\right)^{2}} \\
= & -S_{c c}^{(\mathrm{E})} \frac{M \phi^{2}}{R T_{\mathrm{m}}} L\left[\left(\frac{\partial \Delta g}{\partial c}\right)_{T} \nabla c-\frac{\Delta h}{T} \nabla T\right]_{\mathrm{m}} \frac{\tilde{q}_{x}}{\left(\tilde{q}^{2}+\phi^{2}\right)^{2}},
\end{aligned}
$$

where we have introduced the factor $S_{c c}^{(\mathrm{E})}$ and the scaled inverse penetration depth $\phi$, to facilitate the comparison. Now, we can compare numerically the non-equilibrium enhancements due to mode coupling and due to inhomogeneities by evaluating the (absolute value of the) ratios of the dimensionless prefactors in Eqs. (53) and (54) to (half) $\tilde{S}_{c c}^{(\mathrm{NE})}$ given by Eq. (46). Neglecting the term cubic in the gradient in Eq. (53), we obtain for these ratios,

$$
\begin{gathered}
\frac{-\phi^{2} v D_{0}}{(\nabla c)_{\mathrm{m}} L^{3}} \frac{\left(\frac{\partial^{2} \Delta g}{\partial c^{2}}\right)_{T, \mathrm{~m}}}{\left(\frac{\partial \Delta g}{\partial c}\right)_{T, \mathrm{~m}}^{2}}\left\{1-\frac{M}{R T} \frac{\left(\frac{\partial \Delta g}{\partial c}\right)_{T}^{2}}{\left(\frac{\partial^{2} \Delta g}{\partial c^{2}}\right)_{T}}\right. \\
\left.+\frac{\left[\frac{M \Delta h}{R T} \frac{\partial \Delta g}{\partial c}-\frac{\partial \Delta h}{\partial c}\right]}{T\left(\frac{\partial^{2} \Delta g}{\partial c^{2}}\right)_{T}} \frac{\nabla T}{\nabla c}\right\}_{\mathrm{m}}, \\
\frac{\phi^{2} M v_{0} D_{0}}{L^{3} R T_{\mathrm{m}}(\nabla c)_{\mathrm{m}}}\left[1-\frac{\Delta h}{T}\left(\frac{\partial \Delta g}{\partial c}\right)_{T}^{-1} \frac{\nabla T}{\nabla c}\right]_{\mathrm{m}},
\end{gathered}
$$

where absolute values for the concentration gradients in the denominator are to be taken. The dimensionless numbers (55) turn out to be negligibly small. It is simpler to analyze the second dimensionless ratio (55b), corresponding to inhomogeneously correlated noise. In this case, one finds the product $v_{0} D_{0}$ in the numerator, that is around $10^{-8} \mathrm{~cm}^{4} \mathrm{~s}^{-2}$ for ordinary liquid mixtures, while in the denominator $R T_{\mathrm{m}}$ appears, which in cgs units is of the order of $10^{9}$. Therefore, the second dimensionless ratio in Eq. (55b) is of the order of $10^{-15}$, assuming that the rest of the quantities are of order unity. The first dimensionless ratio in Eq. (55a), corresponding to the inhomogeneous concentration gradient, is somewhat more difficult to analyze because of the presence of the chemical-potential derivatives. Anyway, if we assume that the two derivatives are more or less of the same order, about $R T_{\mathrm{m}} / M$, we still find a negligibly small ratio, of the order of the Eq. (55b) case. In conclusion, the non-equilibrium effects due to inhomogeneities on concentration fluctuations in a reacting binary mixture are completely negligible as compared to mode coupling. We note that a similar situation appears in a simple fluid subjected to a temperature gradient, as analyzed in Ref. 32.

\section{CONCLUSIONS}

In summary, we find that the physical features of the static structure factor are very similar to those found previously ${ }^{13}$ for reactions close to equilibrium. Just as for non-equilibrium fluctuations close to equilibrium, we again find an enhancement of the intensity of the concentration fluctuations in the presence of a temperature gradient. The non-equilibrium concentration fluctuations are in both cases spatially long ranged, with an intensity depending on the wave number $q$. The intensity exhibits a crossover from a $\propto q^{-4}$ to a $\propto q^{-2}$ at wave numbers around $\tilde{q}_{\mathrm{CO}}$ defined by Eq. (47), see also Fig. 3. For wavelengths smaller than the penetration depth the intensity of the non-equilibrium fluctuations varies as $q^{-4}$ just as for the intensity of non-equilibrium 
fluctuations in fluids in the absence of a chemical reaction. ${ }^{7}$ Thus, in a sense, fluctuations with wavelengths smaller than the penetration depth are not affected by the chemical reaction. On the other hand, the intensity of fluctuations with wavelengths larger than the penetration depth varies as $q^{-2}$ caused by the presence of the chemical reaction. ${ }^{7,21,44}$

Fluctuations in chemically reacting mixtures can be probed experimentally by light scattering ${ }^{45,46}$ or by fluorescence correlation spectroscopy. ${ }^{47,48}$ As has been demonstrated for mixtures without chemical reactions, ${ }^{49}$ it should be possible to extend these techniques to investigate nonequilibrium states as well. Hence, the change of the dependence of the intensity of the non-equilibrium concentration fluctuations as a function of the wave number $q$ illustrated in Fig. 3 opens the possibility to distinguish between diffusion- or activation-controlled regimes by these experimental techniques. The important conclusion overall is that also in reaction-diffusion systems far from chemical equilibrium the non-equilibrium correlation functions are long ranged. Amplitudes are modified, but the characteristic features remain the same as in the linear regime. ${ }^{13}$

\section{ACKNOWLEDGMENTS}

J.V.S. and J.O.Z. acknowledge support from The Research Council of Norway, under Grant No. 167336/V30 "Transport on a nano-scale; at surfaces and contact lines." J.O.Z. acknowledges financial support from the Spanish Ministry of Science and Innovation (MICINN) through Grant No. FIS2008-03801. I.P. acknowledges financial support from MICINN through Grant No. FIS2008-04386, and from DURSI under Project No. SGR2009-634. I.P. and J.O.Z. further acknowledge joint support from MICINN under Grant No. FIS2008-04403-E.

\section{APPENDIX: DETAILS ON THE STATIONARY SOLUTION}

To evaluate the solution to Eqs. (10) we prefer, for simplicity, to express all equations in terms of the Onsager transport coefficients $L_{i j} .{ }^{24}$ Corresponding expressions in terms of the common transport coefficients, including the various thermodynamic factors, can readily be derived by using Eqs. (5). Thus, we integrate Eq. (10b) to obtain

$$
\frac{1}{T_{0}}=\frac{L_{J q}}{L_{q q}}\left(\frac{\Delta g_{0}}{T_{0}}\right)+C_{0}+C_{1} \frac{x}{L}
$$

where $C_{0}$ and $C_{1}$ are two integration constants to be discussed later. Notice that Eq. (10b) is valid irrespective of whether the kinetics is linear or nonlinear; hence, the same is true for Eq. (A1). Next, by substituting Eq. (A1) into Eq. (10a), we obtain a closed ordinary nonlinear differential equation for the function $\left(\Delta g_{0} / T_{0}\right)$, namely,

$$
\frac{d^{2}}{d x^{2}}\left(\frac{M \Delta g_{0}}{R T_{0}}\right)=\frac{1}{d^{2}}\left[1-\exp \left(-\frac{M \Delta g_{0}}{R T_{0}}\right)\right]
$$

Similar to the case of linear kinetics, ${ }^{13,16,17}$ we have introduced a penetration depth $d$ (units of length) defined by

$$
\begin{aligned}
\frac{1}{d^{2}} & =\frac{L_{r} L_{q q}}{\left(L_{J J} L_{q q}-L_{J q} L_{q J}\right)} \\
& =\frac{L_{r}}{\rho D T}\left(\frac{\partial \Delta g}{\partial c}\right)_{T}\left[1+\frac{\left(\epsilon_{\mathrm{D}}+k_{T} \Delta \tilde{h}\right)^{2}}{\operatorname{Le} \epsilon_{\mathrm{D}}}\right],
\end{aligned}
$$

where Eqs. (5) have been used in the second equality to express $d$ in terms of ordinary transport coefficients and the various thermodynamic factors. Notice that positive dissipation (entropy production) implies that $d^{2}$ is always positive; and that in the large-Lewis-number approximation used in Sec. IV B, the square bracket in the RHS of Eq. (A3) reduces to unity. Next, we integrate the differential equation (A2) once, obtaining

$$
\frac{d}{d x} \hat{g}=\frac{1}{\sqrt{2} d} \sqrt{C_{2}+\hat{g}+\exp (-\hat{g})},
$$

where $C_{2}$ is a new integration constant and where we adopted the notation $\hat{g}=M \Delta g_{0} / R T_{0}$ to simplify the resulting expression. Next, separating variables we finally obtain

$$
\frac{x}{d}=\sqrt{2} \int_{\hat{g}_{0}}^{\hat{g}(x)} \frac{d u}{\sqrt{C_{2}+u+\exp (-u)}},
$$

with $\hat{g}_{0}$ a fourth integration constant, to be identified with $\hat{g}(x)$ at $x=0$. It is important to note that the linear-kinetics problem can be solved by the same procedure outlined here. In that case $1+\frac{1}{2} u^{2}$ appears instead of $u+\exp (-u)$ in the denominator of Eq. (A5), and the resulting integral can be performed analytically $^{13,16,17}$ and solved for $\hat{g}(x)$. This is not possible for the nonlinear Kramers kinetics case. However, for the discussion of the features of the solution required here, explicit integration of the nonlinear steady-state solution, Eq. (A5), is actually not needed.

What is needed, though, is to evaluate the various integration constants $\left(C_{0}, C_{1}, C_{2}\right.$ and $\left.\hat{g}_{0}\right)$, so that the stationary solution satisfies the four relevant boundary conditions, i.e., impervious walls maintained at different temperatures. Impervious walls require vanishing of diffusion flow $J_{0}$ (including thermal diffusion) at both $x=0$ and $x=L$. The stationary diffusion flow is

$$
\begin{aligned}
J_{0} & =L_{J q} \frac{d}{d x}\left(\frac{1}{T_{0}}\right)-L_{J J} \frac{d}{d x}\left(\frac{\Delta g_{0}}{T_{0}}\right) \\
& =\frac{-R}{\sqrt{2} M d}\left(L_{J J}-\frac{L_{J q}^{2}}{L_{q q}}\right) \sqrt{C_{2}+\hat{g}+\exp (-\hat{g})}+L_{J q} \frac{C_{1}}{L},
\end{aligned}
$$

where, in the second line, we have just substituted Eqs. (A1) and (A4) for the gradients of the thermal properties. Equation (A6) is an analytic relation between the local values of the stationary diffusive flow $J_{0}(x)$ and of the affinity $\hat{g}(x)$. The boundary condition $J_{0}(0)=0$ gives a relationship between three of the integration constants, namely,

$$
C_{1}=\frac{L}{L_{J q}} \frac{R L_{r} d}{\sqrt{2} M} \sqrt{C_{2}+\hat{g}_{0}+\exp \left(-\hat{g}_{0}\right)},
$$


where we use again the definition of the penetration depth $d$ to simplify the resulting expression. We note for future use that the sign of the prefactor in Eq. (A7) depends on the sign of the cross coefficient $L_{J q}$. Next, substitution of Eq. (A7) into Eq. (A6) allows us to express the diffusive flow as

$$
\begin{aligned}
J_{0}= & -\frac{R L_{r} d}{\sqrt{2} M}\left[\sqrt{C_{2}+\hat{g}+\exp (-\hat{g})}\right. \\
& \left.-\sqrt{C_{2}+\hat{g}_{0}+\exp \left(-\hat{g}_{0}\right)}\right] .
\end{aligned}
$$

Because of impervious walls, diffusion has to vanish also at $x=L$. Imposing this condition to Eq. (A8), we get

$$
\hat{g}_{L}+\exp \left(-\hat{g}_{L}\right)=\hat{g}_{0}+\exp \left(-\hat{g}_{0}\right),
$$

with $\hat{g}_{L}=\hat{g}(L)$. In addition to the trivial solution $\hat{g}_{L}=\hat{g}_{0}$, Eq. (A9) has an extra solution for $\hat{g}_{L}$, that can be expressed in terms of the Lambert $W$ function and the value of $\hat{g}_{0}$. If $\hat{g}_{0}$ is negative then the non-trivial $\hat{g}_{L}$ is positive, and vice versa. An interesting fact is that for given $\hat{g}_{0}$, the two boundary conditions for the diffusive flow can be verified independently of the value of the constant $C_{2}$.

Next, and to finalize the specification of the steady-state solution, we have to satisfy boundary conditions for the (inverse) temperature. From Eq. (A1), we readily obtain

$$
C_{0}=\frac{1}{T_{0}}-\frac{R L_{J q}}{M L_{q q}} \hat{g}_{0}, \quad C_{1}=\frac{1}{T_{L}}-\frac{1}{T_{0}}-\frac{R L_{J q}}{M L_{q q}}\left(\hat{g}_{L}-\hat{g}_{0}\right) .
$$

By combining the second part of Eqs. (A10) with Eq. (A7), while taking into account Eq. (A9), one can solve for $C_{2}\left(\hat{g}_{0}\right)$. Then, from Eq. (A5), a single equation for the integration constant $\hat{g}_{0}$ is obtained, namely,

$$
\phi \equiv \frac{L}{d}=\sqrt{2} \int_{\hat{g}_{0}}^{\hat{g}_{L}} \frac{d u}{\sqrt{C_{2}+u+\exp (-u)}} .
$$

This procedure, although a bit complicated, allows for unique determination of the four integration constants and, thus, for a complete solution of the nonlinear problem. We have verified by numerical tests that Eq. (A11) can indeed be solved for $\hat{g}_{0}$. The parameter $\phi$ controls the linearity of the stationary profiles: $\hat{g}(x)$ and $T_{0}(x)$. If $\phi \gg 1$, implying $d \ll L$ or a very fast chemical reaction (see Eq. (A3)), profiles are highly nonlinear. If $\phi \ll 1$ or a very slow chemical reaction, profiles are almost linear as if there were no chemical reaction at all.

In summary, to obtain the solution to Eqs. (10), one must first determine the integration constants $\hat{g}_{0}$ and $\hat{g}_{L}$ by numerically solving Eqs. (A9) and (A11) simultaneously, with $C_{2}\left(\hat{g}_{0}\right)$ given by Eqs. (A7) and (A10). These affinity values at the bounding plates depend on the temperatures $T_{0}$ and $T_{L}$, and the three problem parameters: $\phi$, the prefactor in Eqs. (A10),

$$
a=\frac{R L_{J q}}{M L_{q q}}=\frac{1}{k_{T} T}\left(\frac{\partial \hat{g}}{\partial c}\right)_{T}^{-1} \frac{\epsilon_{\mathrm{D}}\left(\epsilon_{\mathrm{D}}+k_{T} \Delta \tilde{h}\right)}{\operatorname{Le} \epsilon_{\mathrm{D}}+\left(\epsilon_{\mathrm{D}}+k_{T} \Delta \tilde{h}\right)^{2}},
$$

and the prefactor in Eq. (A7),

$$
\begin{aligned}
b & =\frac{L}{L_{J q}} \frac{R L_{r} d}{\sqrt{2} M} \\
& =\frac{\frac{1}{\sqrt{2} k_{T} T}\left(\frac{\partial \hat{g}}{\partial c}\right)_{T}^{-1} \operatorname{Le} \epsilon_{\mathrm{D}}^{2} \phi}{\left(\epsilon_{\mathrm{D}}+k_{T} \Delta \tilde{h}\right)\left[\operatorname{Le} \epsilon_{\mathrm{D}}+\left(\epsilon_{\mathrm{D}}+k_{T} \Delta \tilde{h}\right)^{2}\right]},
\end{aligned}
$$

the last two having dimensions of inverse temperature. Note that a large value of $b / a \phi$ is indicative of a large Lewis number. Once the interval of affinity variation is obtained, diffusion and temperature profiles, $J_{0}(x)$ and $T_{0}(x)$, can be plotted by obtaining the ordinates from Eq. (A5) and the corresponding abscissa from Eqs. (A1) and (A8), respectively.

There is a technical detail that is worth discussing. A condition for the consistency of the solution is that $C_{2}+\hat{g}$ $+\exp (-\hat{g})>0$ for all $\hat{g} \in\left\{\hat{g}_{0}, \hat{g}_{L}\right\}$. This condition can be verified, without loss of generality, by the following scheme: First, $\hat{g}_{0}$ is chosen as the negative solution to Eq. (A8), so that $\hat{g}_{L}-\hat{g}_{0}>0$. Later, the location of the hot temperature is set at $x=0$ or at $x=L$ looking for sign consistency between Eqs. (A7) and (A10). Consistency, or whether to identify the hot temperature with $T_{0}$ or $T_{L}$, depends on the sign of the cross coefficient $L_{J q}$. If $L_{J q}<0$ the sign of $C_{1}$ as given by Eq. (A7) is negative. Hence, in general, we have to choose $T_{L}>T_{0}$ for the sign of $C_{1}$ as given by the second of Eqs. (A10) to be also negative. The reverse is true also in general. ${ }^{50}$ It is obvious that, when mathematically solving the problem we have the freedom to choose the location of the hot temperature, it may happen that the problem solved mathematically is the mirror image of the real problem, but that is not a difficulty. An alternative way of dealing with this consistency issue would be to choose the minus sign of the square root in Eq. (A4). Either way, a consistent solution can be found analytically. This consistency requirement is not only encountered in the present case of nonlinear Kramers kinetics, but also in the case of linear kinetics.

${ }^{1}$ Thermal Nonequilibrium Phenomena in Fluid Mixtures, edited by W. Köhler and S. Wiegand (Springer, Berlin, 2002).

${ }^{2}$ G. F. Froment and K. B. Bischoff, Chemical Reactor Analysis and Design (Wiley, New York, 1990).

${ }^{3}$ A. Stankiewicz and J. A. Moulijn, Re-engineering the Chemical Process Plant. Process Intensification (Marcel Dekker, New York, 2004).

${ }^{4}$ J. Keizer, J. Math. Phys. 18, 1316 (1977).

${ }^{5}$ M. Medina-Noyola and J. Keizer, Physica A 107, 437 (1981).

${ }^{6}$ J. Keizer, Statistical Thermodynamics of Nonequilibrium Processes (Springer, Berlin, 1987), Sec. 6.9.

${ }^{7}$ J. M. Ortiz de Zárate and J. V. Sengers, Hydrodynamic Fluctuations in Fluids and Fluid Mixtures (Elsevier, Amsterdam, 2006).

${ }^{8}$ D. T. Gillespie, J. Phys. Chem. 81, 2340 (1977).

${ }^{9}$ D. T. Gillespie, Annu. Rev. Phys. Chem. 58, 35 (2007).

${ }^{10}$ D. Bernstein, Phys. Rev. E 71, 041103 (2005).

${ }^{11}$ H. Qian, S. Saffarian, and E. L. Elson, Proc. Natl. Acad. Sci. U.S.A. 99, 10376 (2002).

${ }^{12}$ H. Qian, Nonlinearity 24, R19 (2011).

${ }^{13}$ J. M. Ortiz de Zárate, J. V. Sengers, D. Bedeaux, and S. Kjelstrup, J. Chem. Phys. 127, 034501 (2007).

${ }^{14}$ D. Bedeaux, I. Pagonabarraga, J. M. Ortiz de Zárate, J. V. Sengers, and S. Kjelstrup, Phys. Chem. Chem. Phys. 12, 12780 (2010).

${ }^{15}$ H. Kramers, Physica 7, 284 (1940).

${ }^{16} \mathrm{~S}$. R. de Groot and P. Mazur, Non-Equilibrium Thermodynamics (North-Holland, Amsterdam, 1962). 
${ }^{17}$ S. R. de Groot and P. Mazur, Non-Equilibrium Thermodynamics (Dover, London, 1985).

${ }^{18}$ Full compatibility requires $L_{r}$ to be dependent on concentration.

${ }^{19}$ I. Pagonabarraga, A. Pérez-Madrid, and J. M. Rubí, Physica A 237, 205 (1997).

${ }^{20}$ N. G. van Kampen, Stochastic Processes in Physics and Chemistry (North-Holland, Amsterdam, 1982).

${ }^{21}$ C. W. Gardiner, Handbook of Stochastic Methods, 2nd ed. (Springer, Berlin, 1985).

${ }^{22}$ J. Xu, S. Kjelstrup, and D. Bedeaux, Phys. Chem. Chem. Phys. 8, 2017 (2006).

${ }^{23}$ J. Xu, S. Kjelstrup, D. Bedeaux, and J.-M. Simon, Phys. Chem. Chem. Phys. 9, 969 (2007).

${ }^{24}$ J. M. Ortiz de Zárate, D. Bedeaux, I. Pagonabarraga, J. V. Sengers, and S. Kjelstrup, Comptes Rendus Mecanique 339, 287 (2011).

${ }^{25}$ Y. Demirel, Nonequilibrium Thermodynamics. Transport and Rate Processes in Physical and Biological Systems (Elsevier, Amsterdam, 2002).

${ }^{26}$ I. N. Levine, Physical Chemistry, 3rd ed. (McGraw-Hill, New York, 2001).

${ }^{27}$ The fluctuation-dissipation theorem given in Eq. (88) of Bedeaux et al. (Ref. 14) had an incorrect sign.

${ }^{28}$ G. D. C. Kuiken, Thermodynamics of Irreversible Processes (Wiley, Chichester, 1994).

${ }^{29}$ H. N. W. Lekkerkerker and W. G. Laidlaw, Phys. Rev. A 9, 346 (1974).

${ }^{30}$ R. Pérez Cordón and M. G. Velarde, J. Physique (Paris) 36, 591 (1975).

${ }^{31}$ A. M. S. Tremblay, M. Arai, and E. D. Siggia, Phys. Rev. A 23, 1451 (1981).

${ }^{32}$ J. M. Ortiz de Zárate and J. V. Sengers, J. Stat. Phys. 115, 1341 (2004).

${ }^{33}$ M. G. Velarde and R. S. Schechter, Phys. Fluids 15, 1707 (1972).

${ }^{34}$ J. V. Sengers and J. M. Ortiz de Zárate, Thermal Nonequilibrium Phe- nomena in Fluid Mixtures, Lecture Notes in Physics Vol. 584, edited by W. Köhler and S. Wiegand (Springer, Berlin, 2002), pp. 121-145.

${ }^{35}$ J. M. Ortiz de Zárate, J. A. Fornés, and J. V. Sengers, Phys. Rev. E 74, 046305 (2006).

${ }^{36}$ P. N. Segrè, R. W. Gammon, and J. V. Sengers, Phys. Rev. E 47, 1026 (1993).

${ }^{37}$ W. B. Li, K. J. Zhang, J. V. Sengers, R. W. Gammon, and J. M. Ortiz de Zárate, Phys. Rev. Lett. 81, 5580 (1998).

${ }^{38}$ A. P. Fröba, S. Will, and A. Leipertz, Int. J. Thermophys. 21, 603 (2000).

${ }^{39}$ B. Hafskjold and S. K. Ratkje, J. Stat. Phys. 78, 463 (1995).

${ }^{40}$ Y. Demirel, Chem. Eng. Sci. 61, 3379 (2006).

${ }^{41}$ P. N. Segrè, R. W. Gammon, J. V. Sengers, and B. M. Law, Phys. Rev. A 45, 714 (1992).

${ }^{42}$ B. J. Berne and R. Pecora, Dynamic Light Scattering (Wiley, New York, 1976) (Dover, New York, 2000).

${ }^{43}$ There is a misprint in Eq. (59) of Ortiz de Zárate et al. (Ref. 13).

${ }^{44}$ G. Nicolis, A. Amellal, G. Dupont, and M. Mareschal, J. Mol. Liq. 41, 5 (1989).

${ }^{45}$ Y. Yeh and R. N. Keeler, J. Chem. Phys. 51, 1120 (1969).

${ }^{46}$ L. Letamendia, J. P. Yindoula, C. Vaucamps, and G. Nouchi, Phys. Rev. A 41, 3178 (1990).

${ }^{47}$ D. Magde, E. Elson, and W. Webb, Phys. Rev. Lett. 29, 705 (1972).

${ }^{48}$ O. Krichevsky and G. Bonnet, Rep. Prog. Phys. 65, 251 (2002).

${ }^{49}$ W. B. Li, P. N. Segrè, R. W. Gammon, and J. V. Sengers, Physica A 204, 399 (1994).

${ }^{50} \mathrm{We}$ say in general because, in a finer analysis, the selection depends on the magnitude of the parameter $a=R L_{J q} / M L_{q q}\left(\hat{g}_{L}-\hat{g}_{0}\right)$. However, in the large-Lewis-number approximation, $a$ is almost zero and the selection of the hot boundary is always as explained in the main text. 